\title{
The equation of state of quark-gluon matter from lattice QCD with two flavors of twisted mass Wilson fermions
}

\author{
Florian Burger ${ }^{1}$ Ernst-Michael Ilgenfritz, ${ }^{2}$ Maria Paola Lombardo,${ }^{3}$ and Michael Müller-Preussker ${ }^{1}$ \\ (tmf'T collaboration) \\ ${ }^{1}$ Humboldt-Universität zu Berlin, Institut für Physik, 12489 Berlin, Germany \\ ${ }^{2}$ Joint Institute for Nuclear Research, VBLHEP and BLTP, 141980 Dubna, Russia \\ ${ }^{3}$ Istituto Nazionale di Fisica Nucleare, Sezione di Pisa, Largo Pontecorvo 3, \\ I-56100 Pisa, and Laboratori Nazionali di Frascati, INFN, 100044 Frascati, Roma, Italy
}

(Dated: December 21, 2014)

\begin{abstract}
We report on lattice QCD results for the thermodynamic equation of state of quark-gluon matter obtained with $N_{f}=2$ degenerate quark flavors. For the fermion field discretization we are using the Wilson twisted mass prescription. Simulations have been carried out at three values of the bare quark masses corresponding to pion masses of $\sim 360, \sim 430$ and $\sim 640 \mathrm{MeV}$. We highlight the importance of a good control of the lattice cutoff dependence of the trace anomaly which we have studied at several values of the inverse temperature $T^{-1}=a N_{\tau}$ with a time-like lattice extent up to $N_{\tau}=12$. We contrast our results with those of other groups obtained for $N_{f}=0$ and $N_{f}=2+1$. At low temperature we also confront them with hadron resonance gas model predictions for the trace anomaly.
\end{abstract}

PACS numbers: 11.15.Ha, 11.10.Wx, 12.38.Gc

Keywords: Quark-gluon matter, extreme conditions, equation of state, lattice QCD, twisted mass fermions

\section{INTRODUCTION}

Lattice QCD investigations of the (pseudo-) critical behavior of quark and gluon matter at varying temperature have been carried out over many years by several groups employing various improved discretization prescriptions. The special and very demanding task to determine the thermodynamic equation of state (EoS) has reached the physical point, i.e. realistic up-, down- and strange-quark masses. For this aim highly improved staggered fermion discretizations have been employed as reported by the Budapest-Wuppertal group [1, 2] and the HotQCD collaboration [3] (both for $N_{f}=2+1$ dynamical quark degrees of freedom). The staggered fermion approach is most effective from the computational point of view. However, one pays the price of a theoretical uncertainty by applying the rooting trick for the fermionic determinant in order to reduce unwanted (taste) degrees of freedom.

The (improved) Wilson fermion approach is theoretically safe but computationally very demanding and has arrived at the physical point for the zero-temperature case with a corresponding delay [4]. For thermodynamic applications this limit probably will still need some more time. Thermodynamics with improved Wilson quarks has been studied for two quark flavors more than one decade ago by the CP-PACS collaboration [5]. At that time only a very small lattice extent in the Euclidean time direction was feasible $\left(N_{\tau}=4\right.$, or 6$)$. Therefore, the results were strongly influenced by lattice artifacts. The DIK collaboration continued this effort by enlarging $N_{\tau}$ up to 14 lattice units [6, 7]. More recently improved Wilson fermions were studied with $N_{f}=2+1$ on large lattices by the WHOT collaboration [8] and the Budapest-Wuppertal group [9, 10]. Let us also mention attempts to study lattice QCD at nonzero temperature with chirally perfect fermion approaches like the domain wall ansatz 11,13 and overlap fermions [14.

Simulations with a dynamical charm quark, which is expected to be relevant above temperatures of 400 $\mathrm{MeV}$ are also in the course of being performed by the Budapest-Wuppertal [15 and MILC 16 collaborations employing staggered discretizations and by us with Wilson twisted mass quarks in a fixed scale study [17. Recent reviews of the whole subject can be found in $[18,23$.

In addition to the investigations mentioned above, the analysis of the two-flavor model has several reasons of interest. The crossover region and the issue of universality in the chiral limit have been investigated by us with twisted mass Wilson fermions [24, 25, with clover improved fermions in 26] and with $N_{f}=2$ staggered flavors with imaginary chemical potential 27 .

Here, we present our results for the EoS in the two-flavor case with twisted mass fermions. Preliminary results can be found in [25]. From a technical viewpoint, we want to see how twisted mass Wilson fermions perform in the determination of the EoS. Given satisfactory performance we may aim at studying the flavor dependence of the EoS in the critical region by comparing with the quenched and the $N_{f}=2+1$ cases. 
Our main observable is the trace anomaly (also called interaction measure)

$$
I=\epsilon-3 p=T^{5} \frac{\partial}{\partial T}\left(\frac{p}{T^{4}}\right)
$$

related to the partition function by a total derivative with respect to the lattice spacing $a$

$$
I=-\frac{T}{V} \frac{d \ln Z}{d \ln a} .
$$

For the calculation of the temperature dependence of the pressure $p(T)$ and the energy density $\epsilon(T)$ we will employ the integral method (see e. g. 18) according to which the pressure can be evaluated by integrating Eq. (1)

$$
\frac{p}{T^{4}}-\frac{p_{0}}{T_{0}^{4}}=\left.\int_{T_{0}}^{T} d \tau \frac{\epsilon-3 p}{\tau^{5}}\right|_{\mathrm{LCP}}
$$

along a line of constant physics (LCP). The lower bound of the integration has to be set at a sufficiently low temperature $T_{0}$ in such a way that $p_{0}$ is close to zero and can be neglected. Alternatively it may be set from a hadron resonance gas (HRG) model analysis.

In Section II we describe our lattice setup using twisted mass fermions and the tree-level Symanzik improved gauge action followed by the outline of the scale setting prescription in Section III. In Section IV] we are describing the lines of constants of physics along which the temperature integration will be carried out. Since we have redone our scale setting in comparison to our previous work Ref. [24], and since we have added a new (higher) pion mass value we present a new computation of the pseudo-critical temperature in Section V. Section VI provides all details for the computation of the $\beta$-function and prefactors of the fermionic contributions to the trace anomaly. Since we have to subtract $T=0$ results, which are not available for all parameter values discussed, we present the outcome of corresponding interpolations in Section VII. Finally, our results for the trace anomaly, pressure and energy density as functions of the temperature are shown in Sections VIII and IX] In Section $\mathrm{X}$ we show how to use an appropriate modification of the hadron resonance gas model to estimate the low temperature contribution to the pressure. Finally, in Section XI] we contrast our $N_{f}=2$ determination with the quenched and $N_{f}=2+1$ results. In Section XII we will draw the conclusions. In Appendix A we collect the arguments, why one of the contributions to the trace anomaly vanishes in the continuum limit and therefore can be neglected from the beginning. Appendix B lists all tables of simulation parameters and of unrenormalized data for the Polyakov loop and the chiral condensate.

\section{LATTICE TWISTED MASS SETUP}

For the present study of $N_{f}=2$ thermodynamics we have been relying on the twisted mass lattice quark action for two flavors of mass-degenerate quarks

$$
S_{f}^{\mathrm{tm}}[U, \psi, \bar{\psi}]=\sum_{x, y} \bar{\chi}(x)\left(a m \delta_{x, y}+D_{\mathrm{W}}(x, y)[U]+i a \mu \gamma_{5} \tau^{3} \delta_{x, y}\right) \chi(y)
$$

The Wilson discretization of the covariant derivative is given by

$$
D_{\mathrm{W}}(x, y)[U]=4 \delta_{x, y}+\frac{1}{2} \sum_{\mu}\left(1-\gamma_{\mu}\right) U_{\mu}(x) \delta_{y, x+a \hat{\mu}}+\left(1+\gamma_{\mu}\right) U_{\mu}^{\dagger}(x-a \hat{\mu}) \delta_{y, x-a \hat{\mu}}
$$

where the usual Wilson parameter has been put $r \equiv 1$. Via $\kappa \equiv(2 a m+8)^{-1}$ the bare (untwisted) quark mass $m$ is related to the hopping parameter $\kappa$ which has been set to its coupling dependent critical value $\kappa_{c}(\beta)$ as determined by the European Twisted Mass Collaboration (ETMC) 28] and suitably interpolated to the coupling values used in this study [24].

The gauge action is discretized with a tree-level Symanzik improved action

$$
S_{g}^{\mathrm{tlSym}}[U]=\beta\left(c_{0} \sum_{P}\left[1-\frac{1}{3} \operatorname{Re} \operatorname{Tr}\left(U_{P}\right)\right]+c_{1} \sum_{R}\left[1-\frac{1}{3} \operatorname{Re} \operatorname{Tr}\left(U_{R}\right)\right]\right)
$$


with $c_{0}=5 / 3$ and $c_{1}=-1 / 12$ and sums extending over all plaquettes $(P)$ and all planar rectangles $(R)$ attached to each lattice site in positive directions, respectively.

We have simulated three values of the pion mass $m_{\pi} \sim 360, \sim 430$ and $\sim 640 \mathrm{MeV}$, refered to as the B-, C- and D-mass in what follows. In each case several values of $N_{\tau}$ ranging from $N_{\tau}=4$ to $N_{\tau}=12$ have been simulated, see Tables VI, VII, and VIII in Appendix B, The data of the B- and the C-mass have already partly been used in Ref. [24] for the study of the chiral limit of the transition ${ }^{1}$. Preliminary results for the EoS in our setup have been reported in Ref. [25].

\section{SCALE SETTING}

The lattice scale is set using the physical value of the Sommer scale $r_{0}$ determined by ETMC from the nucleon mass in Ref. 29]. For the ETMC generated gauge ensembles the values for $r_{0} / a$ have been published in Ref. [30. Table IV in Appendix B lists the ETMC ensembles we have used and analysed in this work. On additionally generated $T=0$ gauge ensembles, see Table $\mathrm{V}, r_{0} / a$ has been determined from the Euclidean time dependence of the static potential $V\left(x_{4}\right)$, which was extracted from timelike Wilson loops as described in Ref. [30]. The latter have been evaluated on HYP- and APE-smeared configurations. In order to reduce lattice artefacts in the determination of $V\left(x_{4}\right)$ at low values of $\beta$ we employ a tree-level improved definition for the spatial separation as in Ref. 31 for $\beta \leq 3.76$. We perform the chiral limit extrapolation of $r_{0} / a$ for obtaining $r_{\chi} / a$ both with assuming either a linear dependence on the bare quark mass $\mu$ or a pure quadratic dependence without a linear piece. Half of the resulting difference of $r_{\chi}$ is taken as a systematic error and is added to the statistical error of $r_{\chi} / a$. The quadratically extrapolated values have entered our fits as central values. For setting the scale and also for evaluating the $\beta$-function (cf. Section VI we proceed by fitting $r_{\chi} / a$ and the data shifted by $\pm 1 \sigma$ with the following ansatz:

$$
\left(r_{\chi} / a\right)(\beta)=\frac{1+n_{0} R(\beta)^{2}}{d_{0} a_{2 \text { loop }}(\beta)\left(1+d_{1} R(\beta)^{2}\right)}
$$

The ratio $R(\beta) \equiv a_{2 \text { loop }}(\beta) / a_{2 \text { loop }}\left(\beta_{\text {ref }}\right)$ is defined in terms of the two-loop renormalization group formula for the (dimensionless) scale $a_{2 l o o p}(\beta)$ at vanishing fermion mass

$$
a_{2 \text { loop }}(\beta)=\left(\frac{6 \beta_{0}}{\beta}\right)^{-\beta_{1} / 2 \beta_{0}^{2}} \exp \left(-\frac{\beta}{12 \beta_{0}}\right)
$$

with the first two (universal) coefficients of the perturbative $\beta$-function $\beta_{0}=\left(11-2 N_{f} / 3\right) /(4 \pi)^{2}$ and $\beta_{1}=$ $\left(102-\frac{38}{3} N_{f}\right) /(4 \pi)^{4}$. In our analyse we have chosen $\beta_{\text {ref }}=3.9$ for an intermediate reference scale and have checked that the results do not depend on this choice. We add the maximal deviation from the fits to the data of upper and lower error bands from the central fit to the statistical error in quadrature. In order to account for the systematic error associated with the specific choice of a fit function we have performed the fits either setting $d_{1} \equiv 0$ or keeping it as a free parameter in the fit, the former representing our central fit. We propagate the resulting difference of the such fitted $\beta$ dependences of $r_{\chi} / a$ to all subsequent analyses such as the $\beta$-function and other scale dependent quantities.

Allowing for three fit parameters at maximum we use a lower number of parameters compared to Ref. [32, since the functional dependence of $r_{\chi} / a$ on $\beta$ is rather mild, and we have less data points to fit. In Fig. 1 we present the fit which - with twelve data points for $r_{\chi} / a$ at our disposal - yields a good $\chi^{2} /$ dof $=1.6$. The fitted parameters read as follows:

\begin{tabular}{c|c|c|c|c} 
Fit & $n_{0}$ & $d_{0}$ & $d_{1}$ & $\chi^{2} /$ dof \\
\hline 1 & $-0.1096(75)$ & $13.04(12)$ & $\mathbf{0}$ & 1.6 \\
2 & $0.35(30)$ & $12.27(44)$ & $0.62(43)$ & 1.1
\end{tabular}

1 In Ref. 24] an even smaller mass has been considered (called "A-mass"). We have not included it here, since for the EoS required $T=0$ simulations would run into metastable states of the bulk transition occuring at sufficiently small $\beta$. A way out would be to keep at larger $\beta$-values, i.e. to describe the crossover region with a time-like lattice extent $N_{\tau} \geq 14$. Such large lattice sizes go beyond the scope of the present investigation. 

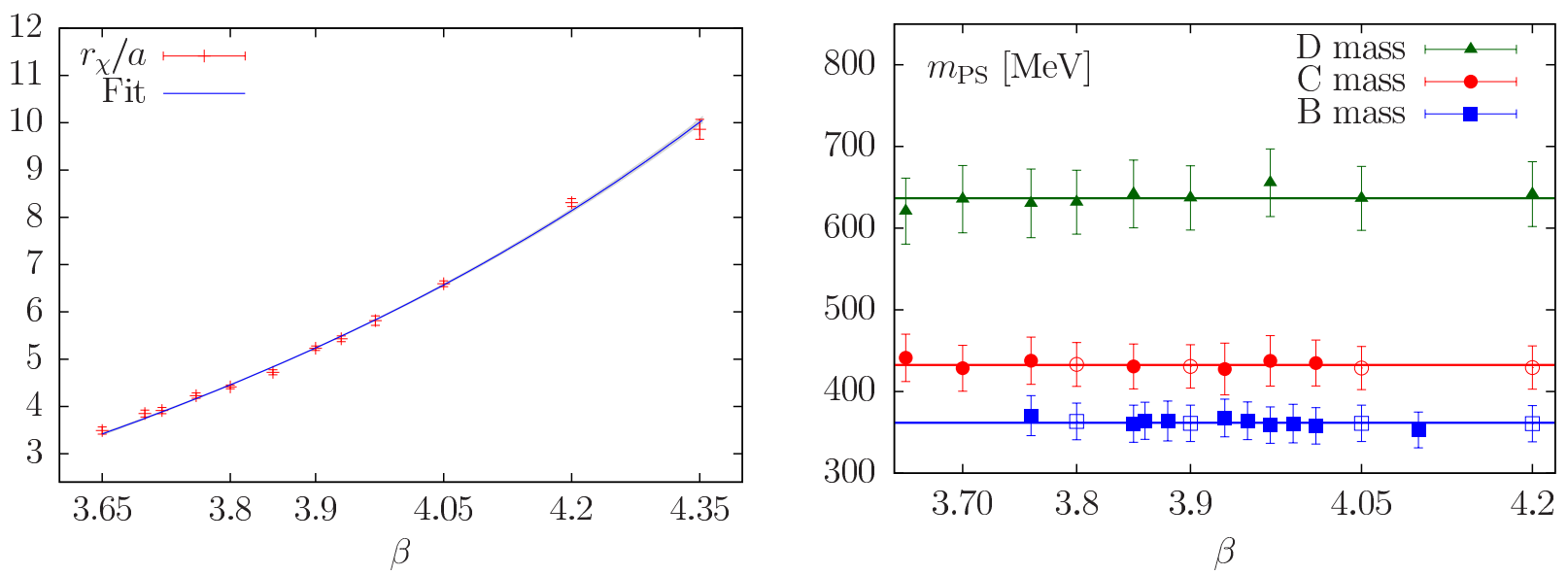

FIG. 1: Left: Chirally extrapolated Sommer scale $r_{\chi} / a$ and a fit using Eq. (7). Right: Charged pion mass in physical units for the three ensembles together with a constant fit over all data points. Open symbols denote data that has been interpolated using ETMC results.

The temperature in physical units is then estimated from the fit using

$$
T(\beta)[\mathrm{MeV}]=\frac{\left(r_{\chi} / a\right)(\beta)}{N_{\tau} r_{0}}
$$

and taking $r_{0}=0.462(28)$ fm from Ref. 29] as input. The uncertainty of the temperature evaluated in this manner is of the order of $4 \%$ throughout the whole temperature range.

\section{LINES OF CONSTANT PHYSICS}

The calculation of the pressure by means of integrating Eq. (3) has to be done on the LCP. To this end we have fixed the mass of the (charged) pion $m_{\mathrm{PS}}$ to three constant values by tuning the bare quark mass by means of the $\beta$-function. The quality of mass tuning is shown in the right panel of Fig.1 1 for the three masses together with a constant fit over the whole range of the coupling. For the B-mass such a figure has already been shown in Ref. [25. - note however that due to the updated value of $r_{0}$ the curve shown there is now slightly shifted. From the fit for the B-, C- and D-masses we obtain the values $m_{\mathrm{PS}}=362(2) \mathrm{MeV}, 433(2) \mathrm{MeV}$ and $637(4)$ $\mathrm{MeV}$, respectively. In the plot we do not show data points obtained at $\beta=4.35$. In this case the box size is very small which leads to an over-estimation of the pseudoscalar masses by about $20 \%$.

\section{THERMAL TRANSITION TEMPERATURE}

Since the scale setting has changed with respect to our previous study in Ref. 24] and one more pion mass has been simulated, by applying the same methods we have conducted a new determination of the pseudo-critical chiral temperature $\left(T_{\chi}\right)$ and - what we conditionally call - the "deconfinement" temperature $\left(T_{\text {deconf }}\right)$.

The chiral temperature $T_{\chi}$ is obtained from fitting Gaussian functions

$$
G(\beta)=a_{G}+b_{G} \cdot \mathrm{e}^{-c_{G}\left(\beta-\beta_{\chi}\right)^{2}}
$$

to the variance of the chiral condensate over configurations, i.e. to (the disconnected part of) the chiral susceptibility, which shows a maximum in the expected crossover temperature region. The fits are performed in the bare coupling, and the pseudo-critical coupling $\beta_{\chi}$ at the center of the Gaussian function is then converted into physical units. In Fig. 2 we show the chiral susceptibility normalised by the squared temperature for the three simulated pion masses as well as for several values of $N_{\tau}$. Note that the data has not been renormalised yet. In addition, in each case we show also the fit curves for the finest discretization of the Euclidean time extent (largest $N_{\tau}$ available). For the smallest mass we can check whether the thermodynamic limit can be considered to be satisfactorily achieved as lattices of a smaller extent $\left(N_{\sigma}=24\right)$ are available for comparison. 
Since - within errors - the susceptibility data for the smaller volume is compatible with the data obtained on the larger volume $\left(N_{\sigma}=32\right)$, we conclude that $T_{\chi}$ is not affected by finite-size scaling effects as one should expect for a crossover phenomenon. We have restricted the Gaussian fits to data obtained in the larger volume.
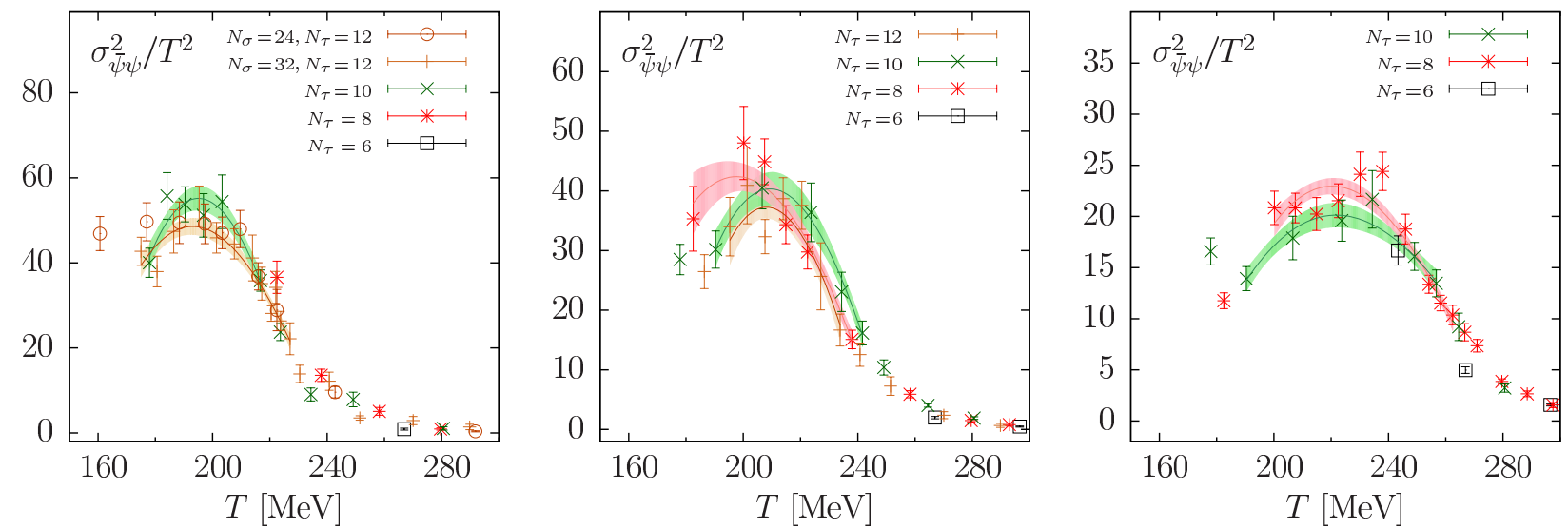

FIG. 2: The disconnected part of the chiral susceptibility in the crossover temperature range together with Gaussian fit curves versus temperature. Left: For the B mass, Middle: C mass, and Right: D mass.

The deconfinement temperature $T_{\text {deconf }}$ is estimated from the renormalised real part of the Polyakov loop $\langle\operatorname{Re}(L)\rangle_{R}$ obtained by multiplicative renormalisation using the static potential $V$ at zero temperature and distance $r_{0}$,

$$
\langle\operatorname{Re}(L)\rangle_{R}=\exp \left(V\left(r_{0}\right) / 2 T\right)\langle\operatorname{Re}(L)\rangle \equiv Z_{L}\langle\operatorname{Re}(L)\rangle .
$$

It is read off from the inflection point of a hyperbolic tangent function

$$
P(T)=a_{P}+b_{P} \cdot \tanh \left(c_{P}\left(T-T_{\text {deconf }}\right)\right)
$$

by fitting the renormalized real part of the Polyakov loop while ignoring the uncertainty in the temperature scale. For $N_{\tau} \geq 8,\langle\operatorname{Re}(L)\rangle_{R}$ shows only small lattice artefacts while they are sizable for $N_{\tau}=4$ and $N_{\tau}=6$ as can be seen in Fig. 3. The fitted values for the deconfinement temperature are listed in the last column of Table [1 The first error indicates the statistical error while the second denotes the uncertainty of the temperature from the scale setting at the fitted $T_{\text {deconf }}$.

In what follows, for the "pseudo-critical temperature" $T_{c}$ we will always use $T_{c} \equiv T_{\chi}$ at the largest $N_{\tau}$ available.
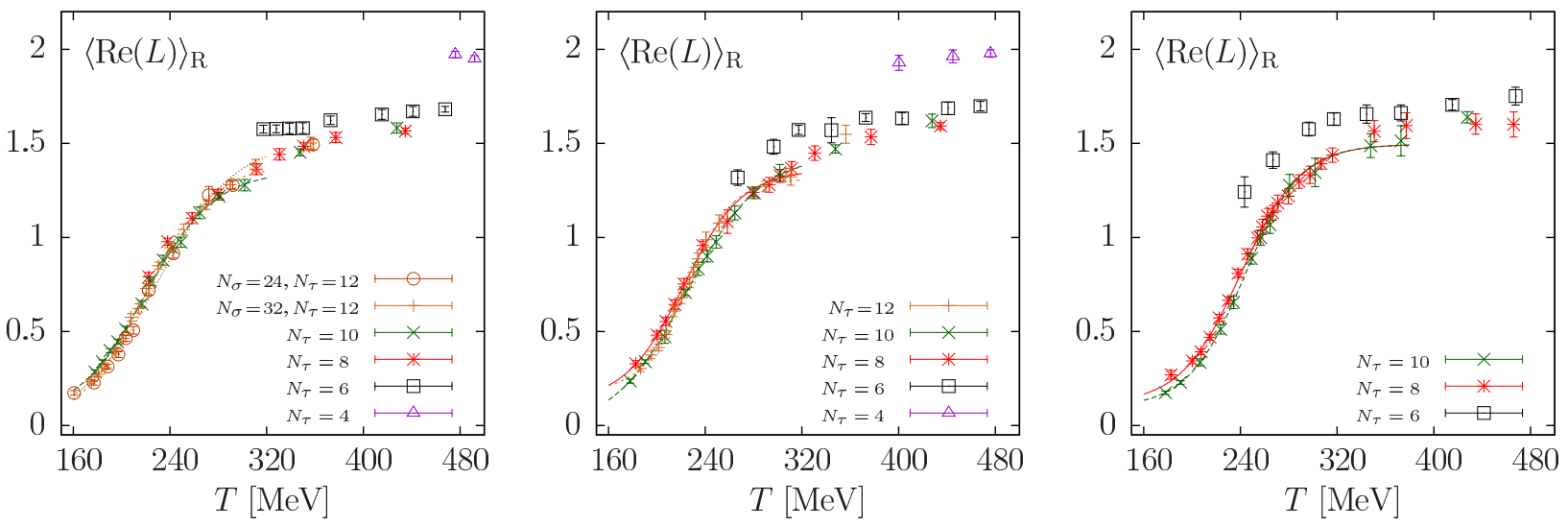

FIG. 3: The renormalised real part of the Polyakov loop versus temperature. Left: For the B mass, Middle: C mass, and Right: D mass. Also shown are fits with $P(T)$ according to Eq. (12) (where evaluated). 


\begin{tabular}{cccccc} 
Ensemble $m_{\mathrm{PS}}[\mathrm{MeV}]$ & $N_{\tau} \times N_{\sigma}^{3}$ & $\beta_{c}$ & $T_{\chi}[\mathrm{MeV}]$ & $T_{\text {deconf }}[\mathrm{MeV}]$ \\
\hline \hline $\mathrm{B}$ & $\sim 360$ & $12 \times 32^{3}$ & $3.92(1)$ & $193(13)$ & $219(3)(14)$ \\
& $12 \times 24^{3}$ & - & - & $223(3)(14)$ \\
& $10 \times 32^{3}$ & $3.82(1)$ & $195(13)$ & $219(4)(14)$ \\
\hline $\mathrm{C}$ & $\sim 430$ & $12 \times 32^{3}$ & $3.97(2)$ & $208(14)$ & $225(3)(14)$ \\
& & $10 \times 32^{3}$ & $3.86(1)$ & $209(14)$ & $225(4)(14)$ \\
& & $8 \times 28^{3}$ & $3.69(3)$ & $198(15)$ & $219(6)(14)$ \\
\hline $\mathrm{D}$ & $\sim 640$ & $10 \times 24^{3}$ & $3.90(3)$ & $229(16)$ & $244(3)(15)$ \\
& & $8 \times 20^{3}$ & $3.75(1)$ & $225(15)$ & $240(2)(15)$
\end{tabular}

TABLE I: List of extracted values for the pseudo-critical temperatures $T_{\chi}$ and $T_{\text {deconf }}$.

\section{TRACE ANOMALY AND SCALE DEPENDENCE OF THE PARTITION FUNCTION}

The computation of the trace anomaly according to Eq. (2) requires to evaluate the derivatives of the partition function with respect to the bare parameters $\kappa, a \mu$ and $\beta$. On the LCP the bare hopping parameter as well as the twisted mass are in turn functions of the gauge coupling.

Employing the following derivatives - which in analogy to the well-known $\beta$-function let us call $B$-functions -

$$
B_{\beta}=a \frac{d \beta}{d a}, \quad B_{\mu}=\frac{1}{(a \mu)} \frac{\partial(a \mu)}{\partial \beta}, \quad B_{\kappa}=\frac{\partial \kappa_{c}}{\partial \beta}, \quad B_{m}=-\frac{1}{(a m)} \frac{1}{\left(2 \kappa_{c}\right)^{2}} B_{\kappa}
$$

as well as the explicit form of our lattice action (Eq. (4) and Eq. (6) ) we arrive at

$$
\begin{aligned}
\frac{I}{T^{4}}=-N_{\tau}^{4} B_{\beta} \frac{1}{N_{\sigma}^{3} N_{\tau}} & \left\{\left\langle\frac{c_{0}}{3} \sum_{P} \operatorname{ReTr} U_{P}\right\rangle_{\mathrm{sub}}+\left\langle\frac{c_{1}}{3} \sum_{R} \operatorname{Re} \operatorname{Tr} U_{R}\right\rangle_{\mathrm{sub}}\right. \\
& \left.-(a \mu) B_{\mu}\left\langle\sum_{x} \bar{\chi}_{x} i \gamma_{5} \tau^{3} \chi_{x}\right\rangle_{\mathrm{sub}}-(a m) B_{m}\left\langle\sum_{x} \bar{\chi}_{x} \chi_{x}\right\rangle_{\mathrm{sub}}\right\} .
\end{aligned}
$$

The expectation values are defined with an implicit subtraction of the corresponding expectation value at $T=0$ in order to render them finite in the ultraviolet:

$$
\langle\ldots\rangle_{\mathrm{sub}} \equiv\langle\ldots\rangle_{T>0}-\langle\ldots\rangle_{T=0}
$$

For later use (see e.g. Appendix B) we will abbreviate the gauge part in form of plaquette and rectangle contributions as

$$
S_{g}=\frac{1}{N_{\tau} N_{\sigma}^{3}}\left(c_{0} \frac{1}{3} \sum_{P} \operatorname{Re} \operatorname{Tr}\left(U_{P}\right)-c_{1} \frac{1}{3} \sum_{R} \operatorname{Re} \operatorname{Tr}\left(U_{R}\right)\right)
$$

and the condensate contribution as

$$
S_{f}=-\frac{1}{N_{\tau} N_{\sigma}^{3}} \sum_{x} \bar{\chi}_{x} i \gamma_{5} \tau^{3} \chi_{x}
$$

Since we are partly relying on the available $T=0$ ETMC data, for taking these subtractions it is necessary to interpolate the data in the mass as well as in the coupling. We shall discuss the strategy in detail in section VII.

The untwisted quark mass related function $B_{m}$ is calculated using ETMC input. To this end we employ the coupling dependence of the critical hopping parameter $\kappa_{c}$, using the prescription indicated in Eq. (13). We have fitted this dependence with a spline ansatz which is shown in Fig. 4. In the low coupling region we have added several further estimates of $\kappa_{c}$. Since we have started the tuning at the largest mass, it was necessary to refine the tuning for smaller masses, such that for couplings below $\beta=3.78$ slightly varying values for the critical hopping parameter have been simulated at a given coupling but at varying twisted mass, while ETMC kept $\kappa_{c}$ fixed in this case. For one value of the coupling $(\beta=3.85, a \mu=0.006)$ we have conducted a check how well 

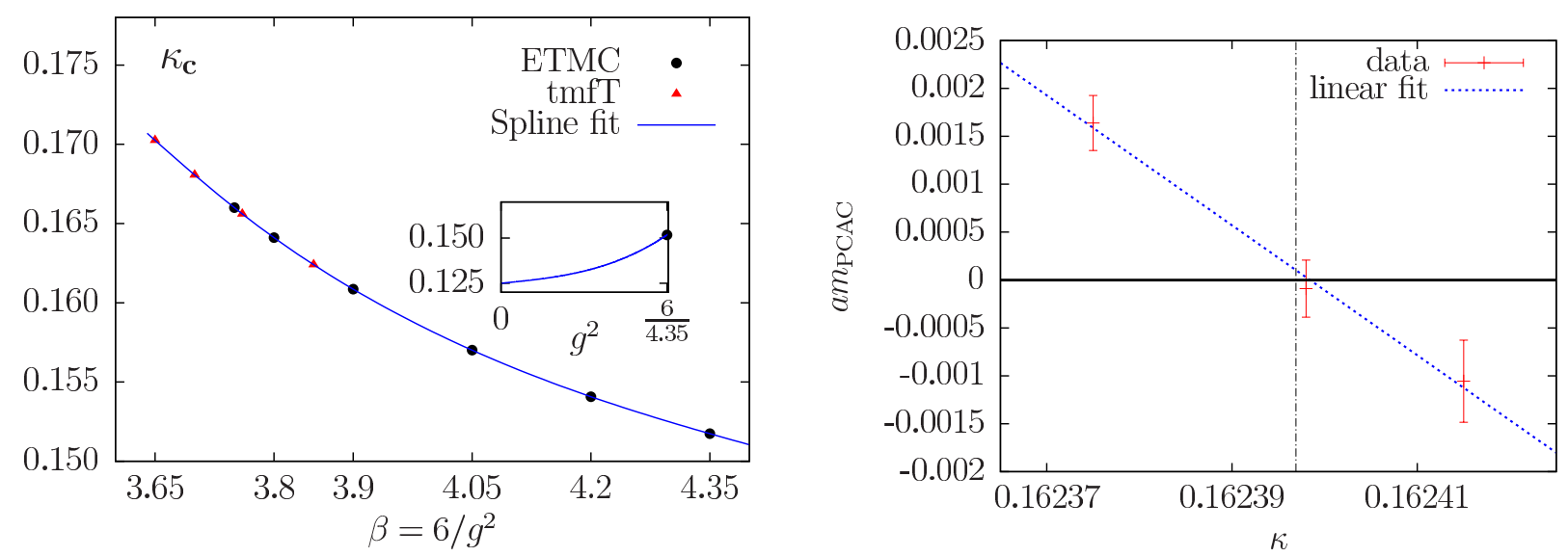

FIG. 4: Left: The dependence of the critical hopping parameter $\kappa_{c}$ on the coupling $\beta$. The curve represents a Padé interpolation. In the inlaid figure we show the interpolation for asymptotically large $\beta=6 / g^{2}$, i. e. small $g^{2}$, where the fit has been constrained to $\kappa_{c}(g \equiv 0)=1 / 8$ representing the asymptotic free limit value.

Right: The PCAC mass as a function of the hopping parameter $\kappa$ around the critical value at $\beta=3.85, a \mu=0.006$. This corresponds to a check of the validity the spline interpolation of $\kappa_{c}$ (see text for details).

the interpolation works in determining $\kappa_{c}$ at an intermediate coupling. To this end we have simulated three values of $\kappa$ in the vicinity of the value predicted by the interpolation and have evaluated the PCAC mass which upon vanishing acts as a criterion for maximal twist [28]. The PCAC mass as well as a linear fit to the data are shown in the right panel of Fig. 4. The critical value of $\kappa$ as predicted from the interpolation is indicated by the vertical line. What can also be seen in the figure is that a slight mistuning leading to a deviation of $\mathcal{O}\left(10^{-3}\right)$ in the PCAC mass results in a value of $\kappa$ being off by $\mathcal{O}\left(10^{-5}\right)$ from its critical value. We thus conclude that the error on the critical $\kappa$ dependence is very small and we thus neglect it in the further analysis, especially for evaluating $B_{m}$ which we take explicitly from the derivative of $\kappa_{c}(\beta)$ using the fitted spline interpolation. For this we have used $\kappa_{c}$ as determined at the lowest mass at given coupling $\beta$.

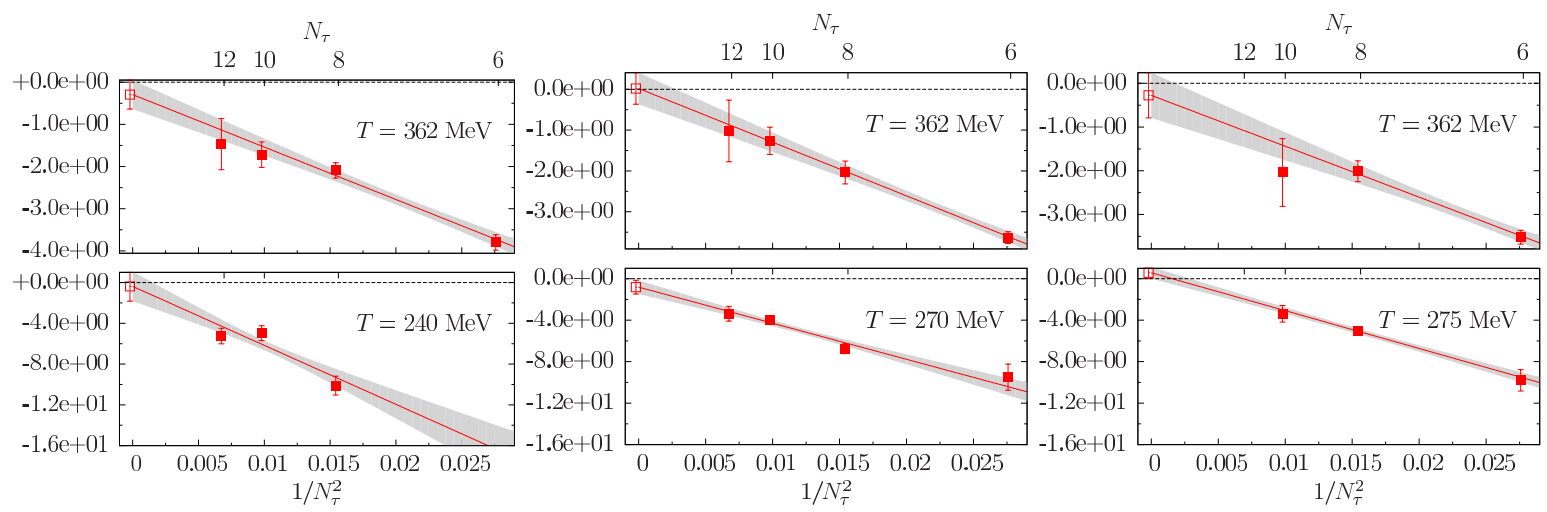

FIG. 5: Left: Check of the continuum limit of the trace anomaly contribution originating from the derivative w. r. t. the untwisted quark mass $m$. We show data for the B mass ensemble at two values of the temperature. Middle: The same for the $\mathrm{C}$ mass. Right: The same for the $\mathrm{D}$ mass.

However, the $m$-derivative term in Eq. (14) containing $B_{m}$ does not contribute in the continuum limit. As will be shown in Appendix A from a Symanzik expansion, the subtracted vacuum expectation value of the operator arising from the $m$-derivative, $\left\langle\sum_{x} \bar{\chi}_{x} \chi_{x}\right\rangle_{\text {sub }}$, is a pure lattice artefact at maximal twist and is vanishing in the continuum limit as $\mathcal{O}\left(a^{2}\right)$. We have checked this numerically by studying the contribution to the trace anomaly from the term in question. Fig. 5 shows the continuum limit of $B_{m}\left\langle\sum_{x} \bar{\chi}_{x} \chi_{x}\right\rangle_{\text {sub }}$. As can be seen in the figure, the extrapolations to $1 / N_{\tau} \rightarrow 0$ of this term are compatible with zero in all studied cases. Therefore, we have not included the contribution of this term upon evaluating Eq. 14 right from the beginning.

The $B$-functions $B_{\beta}$ and $B_{\mu}$ in Eq. (13) are evaluated non-perturbatively from $T=0$ lattice data closely following Ref. [32. In all cases we maintain the correct perturbative behavior of the $B$-functions and incorporate 


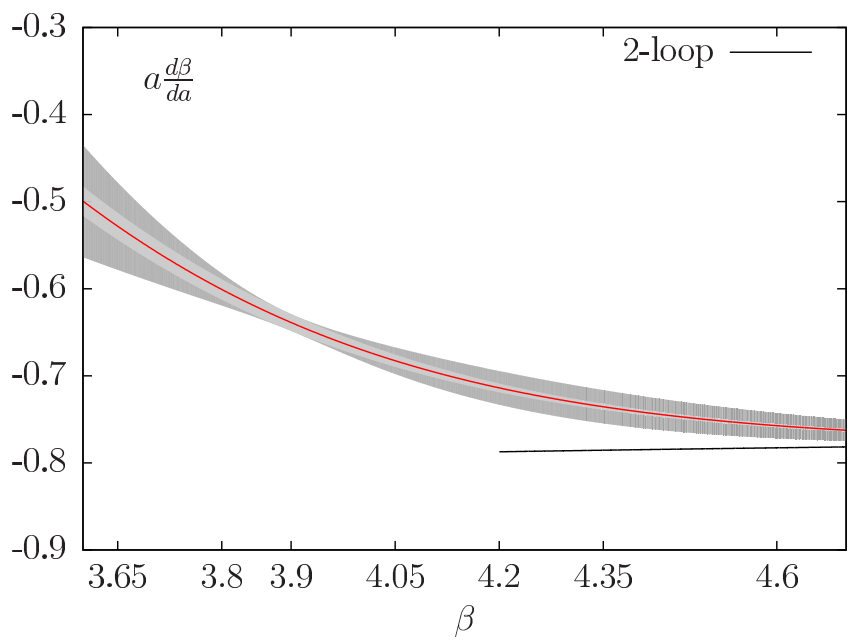

FIG. 6: The $\beta$-function obtained according to Eq. (18) from fitting expression (7) to the chirally extrapolated data of the Sommer scale $r_{\chi} / a$. We also show the perturbative 2-loop expectation at large couplings as obtained from Eq. (8).

it explicitly into fit functions to $T=0$ data. The function $B_{\beta}$, directly related to the non-perturbative $\beta$-function and entering Eq. (14) as a multiplicative factor, is evaluated by means of the following identity in terms of the chirally extrapolated Sommer parameter $r_{\chi} / a$ :

$$
B_{\beta}=\left(a \frac{d \beta}{d a}\right)=-r_{\chi} / a\left(\frac{d r_{\chi} / a}{d \beta}\right)^{-1} .
$$

Using Eq. (18) and the two-loop expression Eq. (8) we obtain the following asymptotic formula valid at large inverse squared coupling $\beta$ :

$$
B_{\beta}(\beta)=-12 \beta_{0}-72 \frac{\beta_{1}}{\beta} .
$$

The interpolation of $B_{\beta}$ determined from the fit is shown in Fig.6 together with the 2-loop perturbative expectation according to Eq. 19 . The grey band in the graph shows the error from the fit which is obtained by means of a bootstrap analysis. The level of the error is of the order of $10 \%$ for low values of $\beta$ and goes down to the $3 \%$ level for higher values.

For the evaluation of the mass renormalization function $B_{\mu}$ we observe

$$
\begin{aligned}
B_{\mu} & =\frac{1}{(a \mu)} \frac{\partial(a \mu)}{\partial \beta} \\
& =\frac{1}{(a \mu)}\left((a \mu) \frac{1}{a} \frac{\partial a}{\partial \beta}+\frac{1}{r_{\chi} / a} \frac{\partial\left(r_{\chi} \mu\right)}{\partial \beta}\right) \\
& =B_{\beta}^{-1}+\frac{1}{r_{\chi} \mu} \frac{\partial\left(r_{\chi} \mu\right)}{\partial \beta}
\end{aligned}
$$

where we have used the fact that $\frac{\partial r_{\chi}}{\partial \beta}=0, r_{\chi}$ being the physical quantity that fixes the scale. Accordingly we fit $\left(r_{\chi} \mu\right)(\beta)$ by the following expression

$$
r_{\chi} \mu=\left(\frac{12 \beta_{0}}{\beta}\right)^{\gamma_{0} / 2 \beta_{0}} P(\beta) .
$$

The first factor gives the leading perturbative $\beta$-dependence of the mass (compare e. g. Ref. [33]) with $\gamma_{0}=$ $1 /\left(2 \pi^{2}\right)$. For the second factor we take a rational ansatz in terms of the ratio $R(\beta)$ as introduced in Eq. (7),

$$
P(\beta)=a_{\mu} \frac{1+b_{\mu} R(\beta)^{2}}{1+c_{\mu} R(\beta)^{2}} .
$$


We employ our fit result of $r_{\chi} / a(\beta)$ for building the product $\left(r_{\chi} \mu\right)(\beta)$. We have fixed $c_{\mu} \equiv 0$ for our main fits and take half the difference to fits with free $c_{\mu}$ but fixed $b_{\mu} \equiv 0$ into account as a systematic error. The fits for the three masses are shown in the left panel of Fig. 7. We obtain reasonable fit results with $\chi^{2} / \mathrm{dof}=0.26,0.27,0.59$ for the $\mathrm{B}, \mathrm{C}$ and $\mathrm{D}$ mass, respectively. We show the result for the combination of $B$-functions $B_{\beta} B_{\mu}$ in the right panel of Fig. 7 and indicate the asymptotic behavior of this quantity $\left(B_{\mu} B_{\beta}=1+\frac{3}{\pi^{3} \beta}\right)$ ) at high values of the coupling. The colored shaded areas correspond to statistical errors and we have visualized the total errors including the systematic fit type related errors by grey bands. The error on $B_{\beta}$ has not been included at this stage of the analysis. It is however accounted for when computing the trace anomaly.
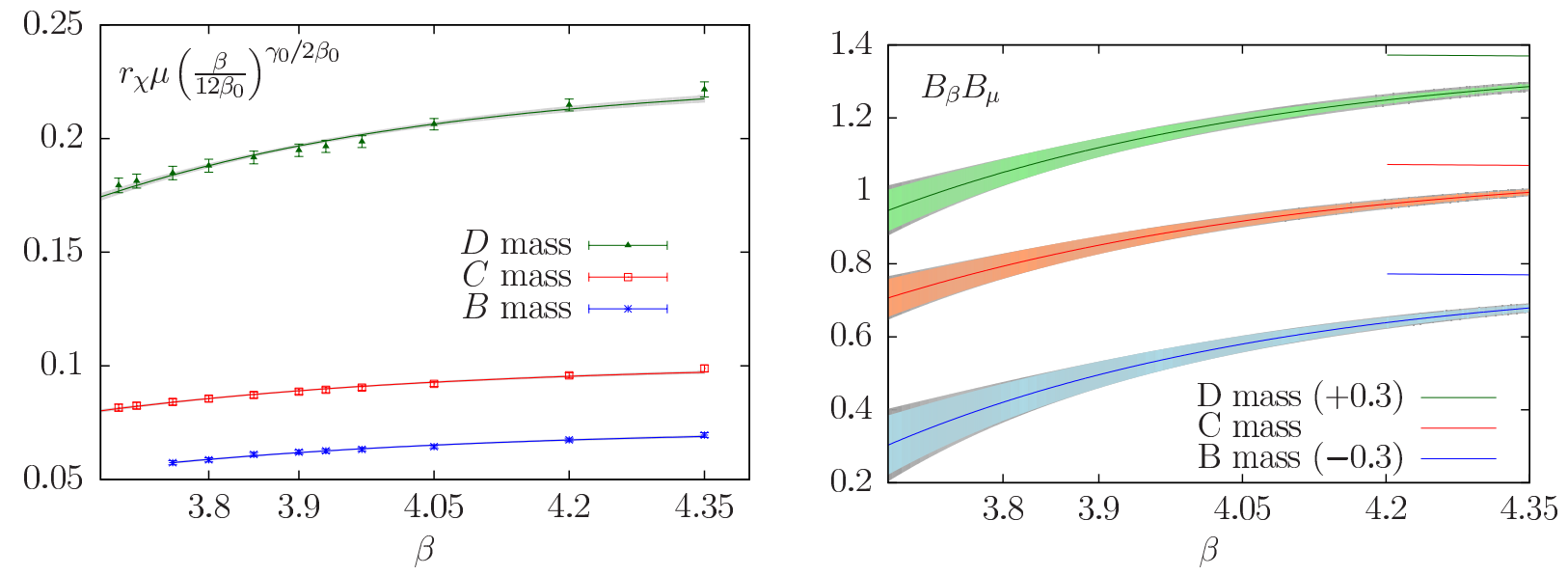

FIG. 7: Left: Fit of $r_{\chi} \mu$ with Eq. 21 for all three masses. Right: Combination of $B$-functions $B_{\beta} B \mu$ for all three masses. The perturbative asymptotic behavior is indicated by the lines at high values of the coupling. The curves for the $\mathrm{B}$ and $\mathrm{D}$ mass have been shifted for better visibility.

\section{INTERPOLATION OF $T=0$ OBSERVABLES}

We have calculated the quantities needed in Eq. (14) for all pairs of values of $\beta$ and $(a \mu)$ that are available from ETMC [30, see Table IV] in Appendix B. Additionally we have substantially increased the $T=0$ data by additional runs, see Table [V] However, not every $T>0$ simulation point has been supplemented by an according $T=0$ simulation. Therefore, we have to interpolate quantities entering Eq. (14) to the precise value of the twisted mass parameter $(a \mu(\beta))$ and the coupling $\beta$ that are used for the finite temperature runs.

The mass dependence of the $T=0$ data points is fitted with cubic spline functions in the bare mass $a \mu$. We use the interpolated values from the fit. This interpolation is only necessary for some values of $\beta$ where the bare masses are not matched to the simulations at $T>0$ (in most cases at values of the couplings that have been studied by ETMC).

The interpolation of these (possibly $(a \mu)$-interpolated) values in the inverse coupling $\beta$ is performed using three types of fit functions in order to study the systematics corresponding to the choice of a specific fit function. Our first choice (further on called type A) is a simple polynomial function with varying degree $d_{p}$

$$
f_{A}^{d_{p}}(\beta)=\sum_{i=0}^{i=d_{p}} c_{i} \beta^{i} .
$$

This ansatz may be extended by splitting the fit up into a low and a high $\beta$ part at a value of $\beta_{\text {cut }}$ leading to a fit type B

$$
f_{B}(\beta)=\left\{\begin{array}{l}
f_{B}^{\text {low }}=f_{A}^{d_{p}}(\beta) \text { if } \beta<\beta_{\text {cut }} \\
f_{B}^{\text {high }} \text { if } \beta>\beta_{\text {cut }}
\end{array},\right.
$$

and we ensure smoothness of the function by an appropriately chosen $f_{B}^{h i g h}$

$$
f_{B}^{h i g h}(\beta)=c_{0}+c_{1}\left(\beta-\beta_{c u t}\right)+\sum_{i=2}^{i=d_{p}} c_{i}\left(\beta-\beta_{c u t}\right)^{i} .
$$




\begin{tabular}{c|cc}
$\chi^{2} /$ dof & Type A & Type B \\
\hline Ensemble B & 1.7 & 1.5 \\
\hline Ensemble C & 2.2 & 1.6 \\
\hline Ensemble D & 3.4 & 1.5
\end{tabular}

TABLE II: Fit quality results for $T=0$ interpolations of $\left\langle S_{g}\right\rangle$ providing the subtraction for the trace anomaly. For fit type A we show the best achieved $\chi^{2} /$ dof with $d_{p}=5$ and for type B with $d_{p}=4$ and varying $\beta_{\text {cut }}$, respectively.

As a third type of fit (type C) function we have considered again a cubic spline function.

The central values for the $T=0$ subtraction of the gauge action contribution to the trace anomaly $\left\langle S_{g}\right\rangle$ are obtained from an average over three fits corresponding to the three fit types A, B and C discussed above. For the former two the quality of the fit is indicated in Table II. These had to be restricted to $\beta \geq 3.7$ for obtaining a good value for $\chi^{2} /$ dof. We therefore restrict the analysis to $\beta \geq 3.7$ disregarding some simulated data at $\beta=3.65$ for the $\mathrm{D}$ and the $\mathrm{C}$ mass. For the $\mathrm{B}$ mass the fits have been conducted in the range $3.76 \leq \beta \leq 4.35$. In the case of fit type $\mathrm{B}$ we have included the best fit result (in terms of $\chi^{2} /$ dof) obtained when varying $\beta_{\text {cut }}$ in Eq. (24). For type A we have restricted ourselves to $d_{p}=5$ since only for this choice the quality of the fit was reasonable. Our final value is obtained from an average over the three fits and taking half the maximal deviation of either of the three fits from the central value into account as a systematic error which is added to the statistical errors as obtained from fit type A in quadrature.

Since the number of available $T=0$ points is very limited above $\beta=4.0$ we had to take special care in order to obtain a reliable interpolation for the inverse coupling $\beta=4.25$ for the B mass and $N_{\tau}=12$. In this case the precision at $T>0$ is good enough to see a $\sim 2 \sigma$ effect on the value of $I / T^{4}$ corresponding to $\beta=4.25$. To this end we have fitted the above fit functions of type A and B with lower number of parameters there and used these interpolations for the subtractions at the inverse coupling parameter values $\beta=4.25$ and $\beta=4.35$. For the $\mathrm{C}$ mass and $N_{\tau}=12$ with less statistics the effect is at the $\sim 1 \sigma$ level only and we stick to the analysis in terms of type $\mathrm{A}, \mathrm{B}$ and $\mathrm{C}$ fits fitted globally to all values of the coupling.

In Fig. 8 we show results of the fits of type A for the three mass values. As from the figures themselves it is impossible to estimate the quality of the fit due to the small errors, we show the residuals of the fits (i. e. the difference of the data and the fit normalised by the corresponding errors) in the lower panels of the figures. Having in mind the reasonable values of $\chi^{2} /$ dof obtained we underline the fact that the different kinds of fits have provided curves which nicely fall on top of each other.

For the fermionic contribution $\left\langle S_{f}\right\rangle$ we used exclusively the fit type $\mathrm{C}$ for subtracting the divergent contribution at $T=0$. The reason is that for the $\mathrm{B}$ ensemble the tuning of the mass $\mu$ has not been done on the same footing for all couplings. While in the near vicinity of the crossover the one-loop $\beta$-function has been used, we have opted for the two-loop $\beta$-function at larger as well as smaller couplings. For $N_{\tau}=10$ the mass has been even tuned only very approximately in the range $3.86 \leq \beta \leq 3.93$ and $a \mu=0.006$ has been set. Since the divergence to be subtracted is of the form $\sim a \mu / a^{3}$, and thus sensitive to the mass, bad fit results can be expected when the precise value of $a \mu(\beta)$ is slightly changed when varying $\beta$. We note however that this slight variation in the way of tuning $a \mu$ does not affect the tuning of the physical pion mass, i. e. the line of constant physics, as was shown in section IV.

\section{TRACE ANOMALY RESULTS}

In this section we present our results for the trace anomaly and the therefrom derived thermodynamic quantities for the B, C and D ensembles. The data is shown for varying $N_{\tau}$ in Fig. 9 where we observe severe lattice artefacts. In order to remedy this large effect we have studied what is known in literature as tree-level improvement Ref. [1].

The starting point for this method are the bosonic and fermionic pressures per degree of freedom in the noninteracting limit which read $p_{B}=\frac{\pi^{2}}{90} T^{4}$ and $p_{F}=\frac{7}{8} \frac{\pi^{2}}{90} T^{4}$, respectively. Upon counting the number of bosonic and fermionic degrees of freedom for $N_{f}=2$ QCD we obtain the Stefan-Boltzmann limit of the pressure as

$$
\frac{p_{\mathrm{SB}}}{T^{4}}=\left(16+\frac{7}{8} \times 24\right) \frac{\pi^{2}}{90} \approx 4.0575 .
$$

On the lattice the free limit pressure $p_{\mathrm{SB}}^{L}$ receives $N_{\tau}$-dependent corrections that vanish in the continuum limit. It has been calculated for the twisted mass action in Ref. 34. Through the mass dependence of the 

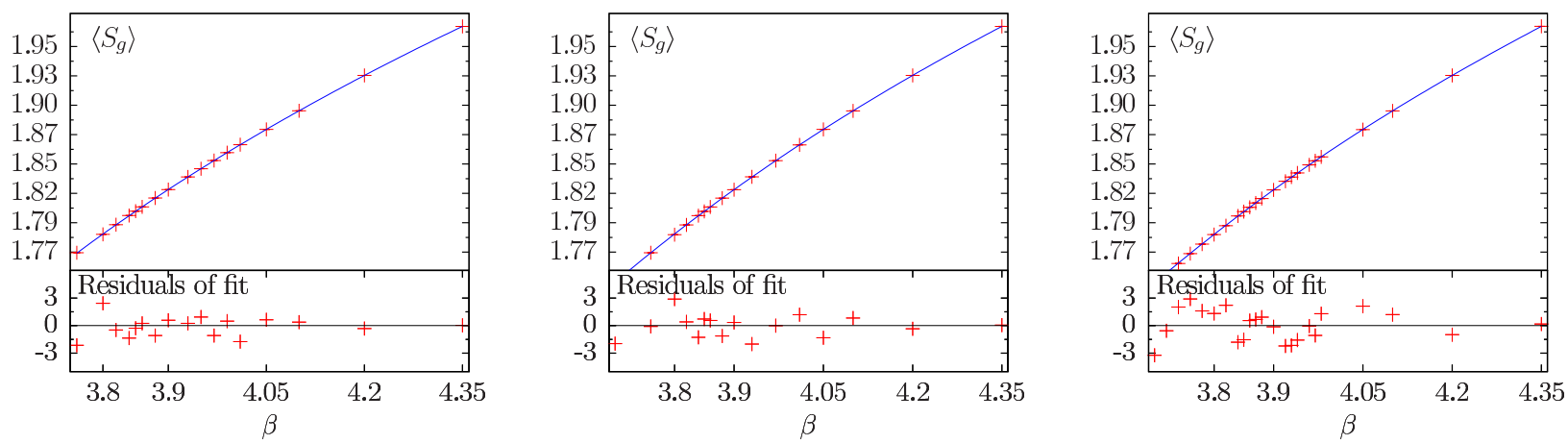

FIG. 8: Interpolation of $(T=0)$ values in $\beta$ for the gauge action contributions to the trace anomaly for (from left to right) the $\mathrm{B}, \mathrm{C}$ and $\mathrm{D}$ mass. We show the outcome of a fit using fit type A with $d_{p}=5$. In each case we show the residuals of the fits in the lower panels in order to illustrate the quality of the interpolation. From left to right the resulting values for $\chi^{2} /$ dof have been $1.7,2.2$ and 3.4 , respectively.
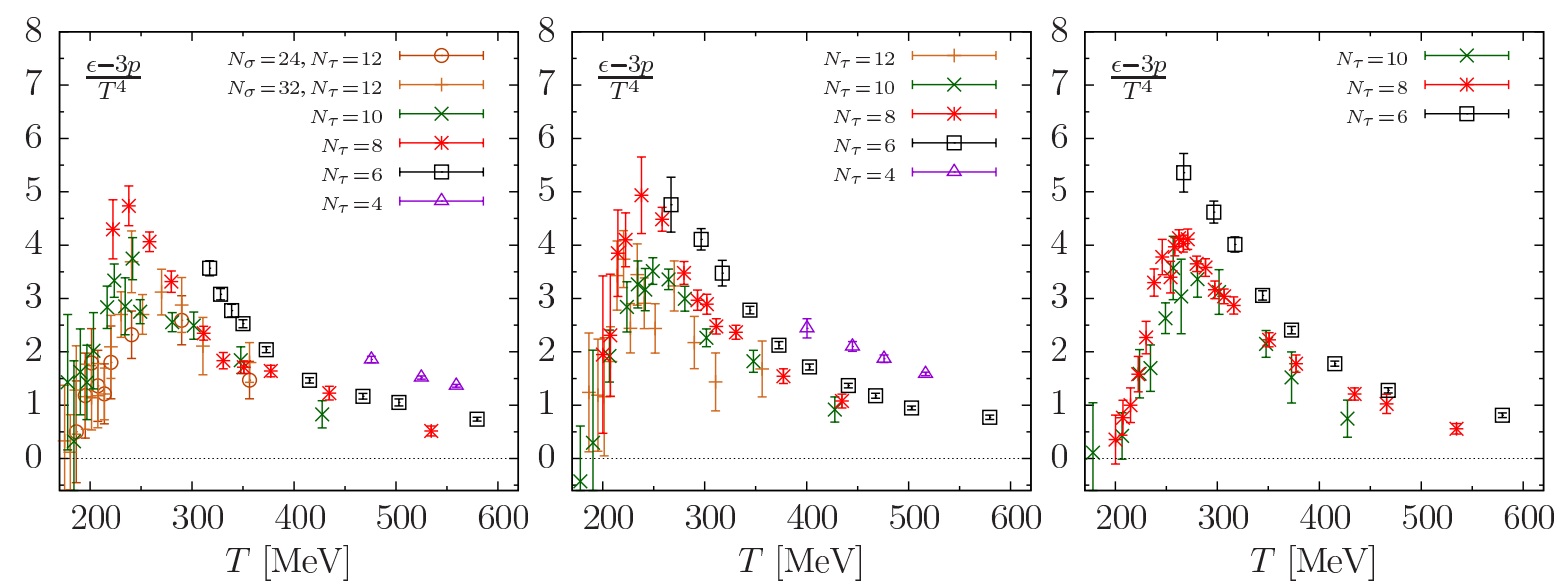

FIG. 9: Left: The trace anomaly for the B mass obtained for different values of the temporal extent $N_{\tau}$. Middle: The same quantity for the $\mathrm{C}$ mass. Right: The same quantity for the D mass. For the B mass the results obtained on the smaller spatial volume are superimposed slightly shifted for better visibility.

fermion propagator, $p_{\mathrm{SB}}^{L}$ as well as $p_{\mathrm{SB}}$ depend in general on the ratio $\frac{m_{R}}{T}$ of renormalised quark mass and temperature. However, this dependence is weak, the change being of the order of below $1 \%$ when varying $m_{R}$ in the ranges of the twisted masses we have simulated. We have used the ratio $p_{\mathrm{SB}}^{L} / p_{\mathrm{SB}}$ to correct the trace anomaly data for its leading cutoff effects.

Together with the corresponding ratio for the tree-level Symanzik improved gauge action that can be found in Ref. $[35]^{2}$ we obtain the following correction factors that are used throughout this work:

$$
\begin{array}{c|ccccc}
N_{\tau} & 4 & 6 & 8 & 10 & 12 \\
\hline \hline p_{\mathrm{SB}}^{L} / p_{\mathrm{SB}} & 2.576 & 1.631 & 1.263 & 1.134 & 1.082
\end{array} .
$$

The tree-level correction of the trace anomaly then amounts to making the following replacement in the whole temperature interval:

$$
\left(\frac{I}{T^{4}}\right) \Rightarrow\left(\frac{I}{T^{4}}\right) /\left(\frac{p_{\mathrm{SB}}^{L}}{p_{\mathrm{SB}}}\right)
$$

\footnotetext{
${ }^{2}$ Since the ratio rapidly approaches unity for increasing $N_{\tau}$ we adopt a value of 1 for $N_{\tau}=12$ which induces a negligible error.
} 


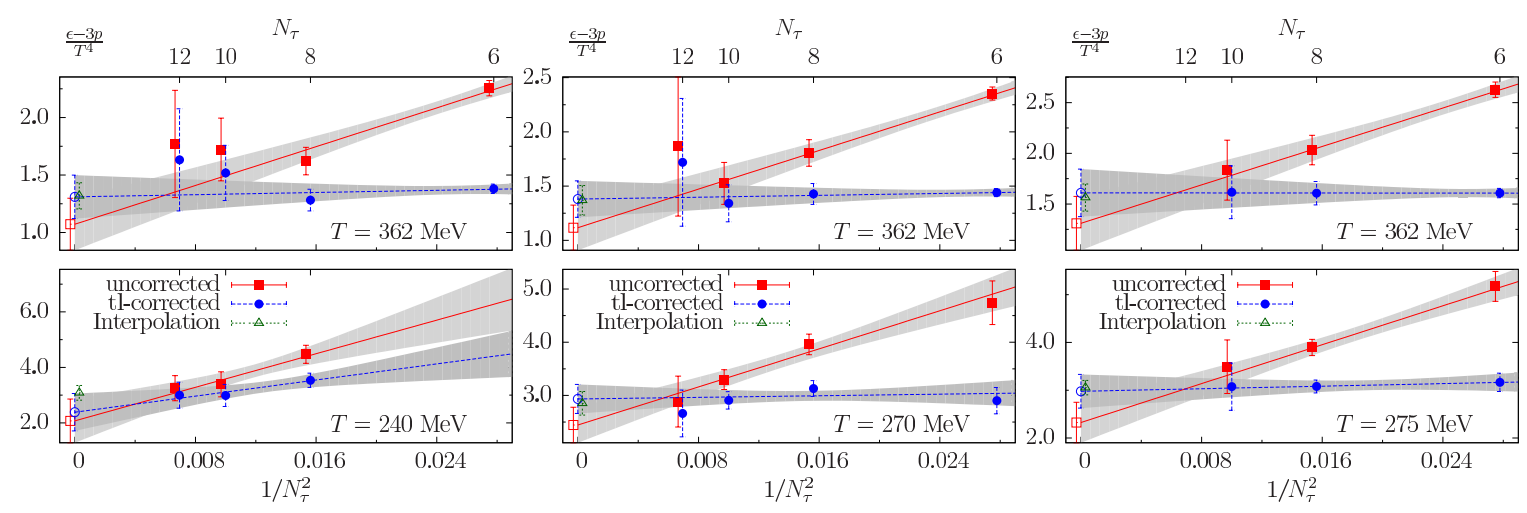

FIG. 10: Continuum limit of the trace anomaly for three masses and for in each case two values of the temperature once with tree-level correction (blue circles and lines) and once without (red squares and lines). We compare the continuum limit results for the two extrapolations with the continuum estimate provided by the global fit Eq. (28) at the same temperature (green triangles).

The tree-level correction of the trace anomaly may be checked by studying the continuum limit of $I / T^{4}$ with and without the correction in place. To this purpose we show in Fig.10 a comparison of two different ways to take the continuum limit of the trace anomaly for the three ensembles. In each case we consider two values of the temperature $T=(240$ and 362$) \mathrm{MeV}, T=(270$ and 362$) \mathrm{MeV}$ and $T=(275$ and 362$) \mathrm{MeV}$ for the $\mathrm{B}, \mathrm{C}$ and $\mathrm{D}$ ensemble, respectively. The smaller temperature was chosen in the range of the maximum of the interaction measure, while the higher temperature is situated in the falling (right) flank. Data for different $N_{\tau}$ was interpolated using a second order polynomial fitted to the four data points closest to the given temperature under investigation. We perform continuum extrapolations linear in $1 / N_{\tau}^{2}$ including $N_{\tau}=12,10,8$ (where possible also $N_{\tau}=6$ ) once with the multiplicative correction (Eq. (27)) in place and once without it. We observe that both procedures lead to compatible continuum limit values matching each other within two standard deviations for the trace anomaly. The correction leads in general to a flatter continuum limit than we observe for the uncorrected data. Apart from $T=240 \mathrm{MeV}$ for the B mass (where no $N_{\tau}=6$ data point is available) the corrected results are even compatible with a flat continuum limit. Moreover, the corrected trace anomaly at the two largest temporal extents $\left(N_{\tau}=8,10\right.$ for the D mass and $N_{\tau}=10,12$ for the B mass $)$ are in all cases compatible with each other within errors.
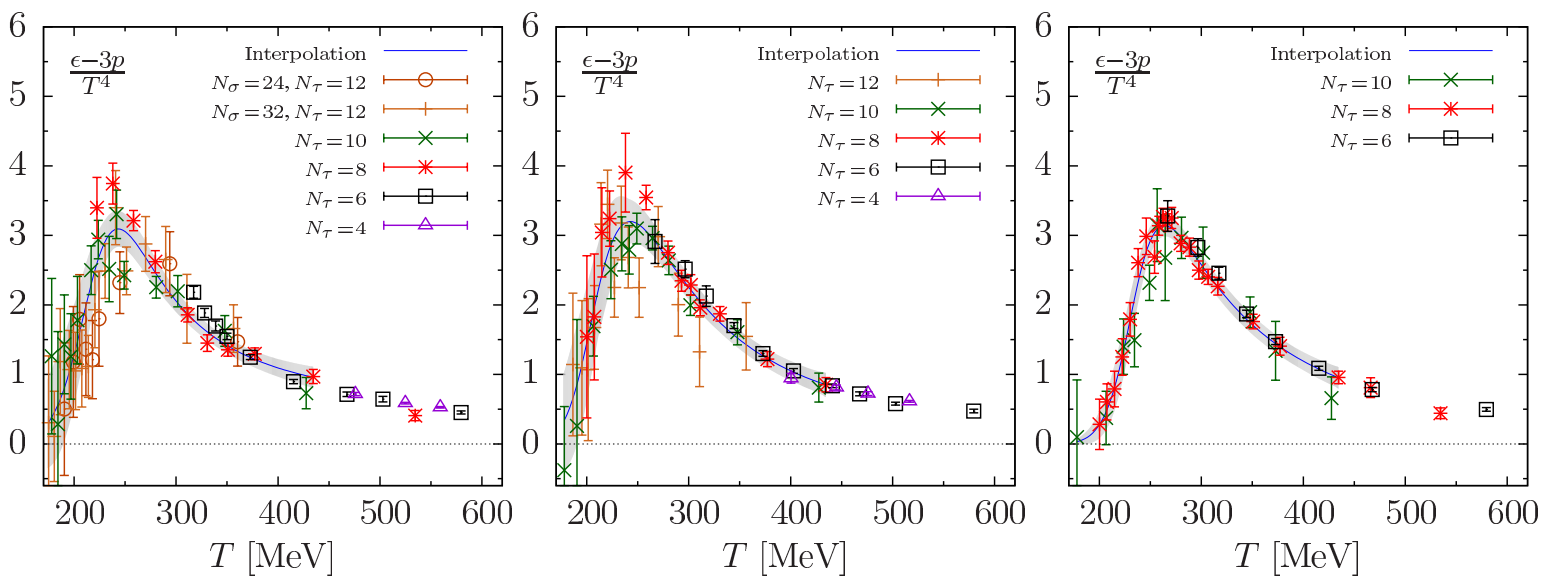

FIG. 11: Left: The tree-level corrected trace anomaly for the B mass obtained for different values of the temporal extent $N_{\tau} . T_{\chi}$ and $T_{\text {deconf }}$ are located at 193 and $219 \mathrm{MeV}$, respectively. Middle: The same quantity for the $\mathrm{C}$ mass with $T_{\chi}$

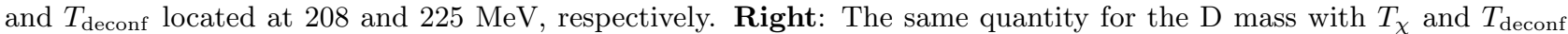
located at 229 and $244 \mathrm{MeV}$, respectively. Also shown is the result of a combined fit of the interpolation formula Eq. (28) to the $N_{\tau}=8,10$ and 12 data $\left(N_{\tau}=6,8\right.$ and 10 in case of the D mass). For the B mass the results obtained on the smaller spatial volume are superimposed slightly shifted for better visibility. This data however has not been included in the fit.

The integration of Eq. (3) is performed by fitting a modified version of an ansatz used in Ref. 1] to the 


\begin{tabular}{|c|c|c|c|c|c|}
\hline Ensemble & \multicolumn{5}{|c|}{ PARAMETERS } \\
\hline \multirow{4}{*}{ B } & $h_{0}$ & $h_{1}$ & $h_{2}$ & $f_{0}$ & $f_{1}$ \\
\hline & $0.20(13)$ & $-4.4(1.4)$ & $4.9(1.8)$ & $0.074(21)$ & $0.9090(3)$ \\
\hline & $f_{2}$ & $g_{1}$ & $g_{2}$ & $T_{0}$ & $\chi^{2} /$ dof \\
\hline & $5.5112(3)$ & $-1.83(8)$ & $0.88(7)$ & $211(4)$ & 1.7 \\
\hline ENSEMBLE & \multicolumn{5}{|c|}{ PARAMETERS } \\
\hline \multirow{4}{*}{$\mathrm{C}$} & $h_{0}$ & $h_{1}$ & $h_{2}$ & $f_{0}$ & $f_{1}$ \\
\hline & $0.03(3)$ & $-8.7(2.5)$ & $6.8(2.8)$ & $0.021(9)$ & $0(3)$ \\
\hline & $f_{2}$ & $g_{1}$ & $g_{2}$ & $T_{0}$ & $\chi^{2} /$ dof \\
\hline & $1(4)$ & $-2.2(2)$ & $1.29(18)$ & $238(2)$ & 1.2 \\
\hline ENSEMBLE & \multicolumn{5}{|c|}{ PARAMETERS } \\
\hline \multirow{4}{*}{$\mathrm{D}$} & $h_{0}$ & $h_{1}$ & $h_{2}$ & $f_{0}$ & $f_{1}$ \\
\hline & $0.05(7)$ & $-5.3(7)$ & $5.3(8)$ & $0.09(2)$ & $1.15421(9)$ \\
\hline & $f_{2}$ & $g_{1}$ & $g_{2}$ & $T_{0}$ & $\chi^{2} /$ dof \\
\hline & $5.73995(7)$ & $-2.2(6)$ & $1.27(6)$ & $268(2)$ & 0.90 \\
\hline
\end{tabular}

TABLE III: Fit parameters obtained from fits of Eq. 28 to the tree-level corrected trace anomaly data of the B, C, and D mass ensembles, respectively.

available lattice data for $\frac{I}{T^{4}}$ (discarding those for $\left.N_{\tau}=4\right)$.

$$
\frac{I}{T^{4}}=\left(1+\frac{a_{2}}{N_{\tau}^{2}}\right) \times \exp \left(-h_{1} \bar{t}-h_{2} \bar{t}^{2}\right) \cdot\left(h_{0}+\frac{f_{0}\left\{\tanh \left(f_{1} \bar{t}+f_{2}\right)\right\}}{1+g_{1} \bar{t}+g_{2} \bar{t}^{2}}\right) .
$$

While in Ref. [1] the normalisation temperature $T_{0}$ in the dimensionless ratio $\bar{t} \equiv T / T_{0}$ has been fixed we let $T_{0}$ vary in the fit. Since we observe large cutoff effects in our trace anomaly results we furthermore include a multiplicative correction term into our fit function incorporating the leading and sub-leading $N_{\tau}$ dependence.

Table III lists the best fit parameters together with errors of fits of the interpolation formula Eq. (28) to the trace anomaly data for the $\mathrm{B}, \mathrm{C}$, and $\mathrm{D}$ ensembles, respectively, after the trace anomaly has been corrected using the tree-level correction Eq. 277). The table also provides the $\chi^{2} /$ dof values of these fits. The interpolation curves are illustrated together with the data corresponding to the three pion mass values in Fig.11.

The error of the interpolation indicated by a grey band in these figures is evaluated as follows. From fits to bootstrap samples of our data we estimate a first error of our interpolation, giving rise to the errors on the fit parameters presented in Table III A second error is obtained by fitting the interpolation function to the data shifted by one standard deviation in the upper and lower directions and measuring the deviation to the fit of the original data. Both errors are then added in quadrature. We have adopted this rather non-standard method because we have observed that the first of these errors (originating from the bootstrap analysis) is very small as compared to the uncertainties of the data themselves. This is especially true for the low temperature region. Thus by considering the pure fit error we would certainly have underestimated the error of the trace anomaly interpolation there.

For the D ensemble we have included $N_{\tau}=10$ and $N_{\tau}=8$ into the fit, while for the $\mathrm{C}$ and B ensemble we fit $N_{\tau}=12,10$ and 8. This approach, which assumes a behavior constant in $N_{\tau}$ towards the continuum limit, is justified by the effectiveness and reliablility of the tree-level correction. The latter effectively superimposes data from different $N_{\tau}$ as can be seen from Fig.11. We have explicitly checked the outcome of our global fits for two values of the temperature in Fig. 10 , where we compare standard continuum extrapolations in $1 / N_{\tau}^{2}$ for data with and without tree-level correction to the continuum estimate provided by this fit. In all cases we find compatible continuum results.

We conclude this paragraph with a discussion of finite size effects. At $T>0$ the thermodynamic limit has been studied for the smallest pion mass. We have evaluated the trace anomaly for $N_{\tau}=12$ reducing the spatial extent from $N_{\sigma}=32$ to $N_{\sigma}=24$. As can be seen from Fig.11 the results on the smaller volume are compatible (within the large errors) with the result obtained in the larger volume. 


\section{PRESSURE AND ENERGY DENSITY}

From the fitted interpolation of the interaction measure Eq. $(28)$ it is straightforward to calculate the pressure by performing a numerical integration starting in all cases from the lowest available data point of $I / T^{4}$ where we set the pressure equal to zero. In other words we set $p_{0}=0$ in Eq. (3) with $T_{0}$ being our smallest temperature $T=174 \mathrm{MeV}(T=177 \mathrm{MeV})$ for the $\mathrm{B}(\mathrm{C}$ and $\mathrm{D})$ mass. In section $\mathrm{X}$ we try to estimate $p_{0}$ from a comparison of our $N_{f}=2$ lattice data at unphysically high masses to adapted HRG models.

In this way we obtain the pressure (and the energy density from adding three times the pressure to $I$ ) for all temperatures in the temperature interval covered by our simulations. We do not restrict ourselves to the points in $T$ where we actually have lattice data, but rather give the corresponding error channels for all upper integration bounds. This seems to us the most natural choice as we have included into the interpolation fits to $I / T^{4}$ data from several values of $N_{\tau}$. In Fig. 12 we show our results for the pressure $(3 p)$ as well as the energy density $(\epsilon)$ as a function of the temperature. At the top of the three panels in this figure we also mark the temperature in units of the pseudo-critical temperature $T_{c} \equiv T_{\chi}$ the latter determined from the maximum of the chiral susceptibility in each mass case. We used the estimate originating from the largest $N_{\tau}$ in all cases.

The energy density features a sharp rise around $T_{c}$ signalling the transition into the quark-gluon plasma regime. At temperatures of about $\sim 1.3 T_{c}$ however, the increase has stopped and we observe an almost constant behavior up to the largest temperatures considered. This feature is also observed by other groups, cf. Ref. 1, 5, 36. At large temperatures we can confront pressure and energy density to the ideal gas StefanBoltzmann pressure (Eq. (26)), which is indicated by the black arrow to the right of the figures. At our largest accessible temperature corresponding to $T / T_{c} \sim 2$ our computed energy density attains roughly half of its expected asymptotic Stefan-Boltzmann limit value.

Another observable of phenomenological interest derivable from the basic bulk thermodynamic quantities $p$ and $\epsilon$ is the velocity of sound of the hot medium which is defined as the derivative of the pressure with respect to the energy density

$$
c_{s}^{2}=\frac{d p}{d \epsilon}
$$

and may be calculated from the ratio $p / \epsilon$ by means of the following identity [36]:

$$
\frac{d p}{d \epsilon}=\epsilon \frac{d(p / \epsilon)}{d \epsilon}+\frac{p}{\epsilon}
$$
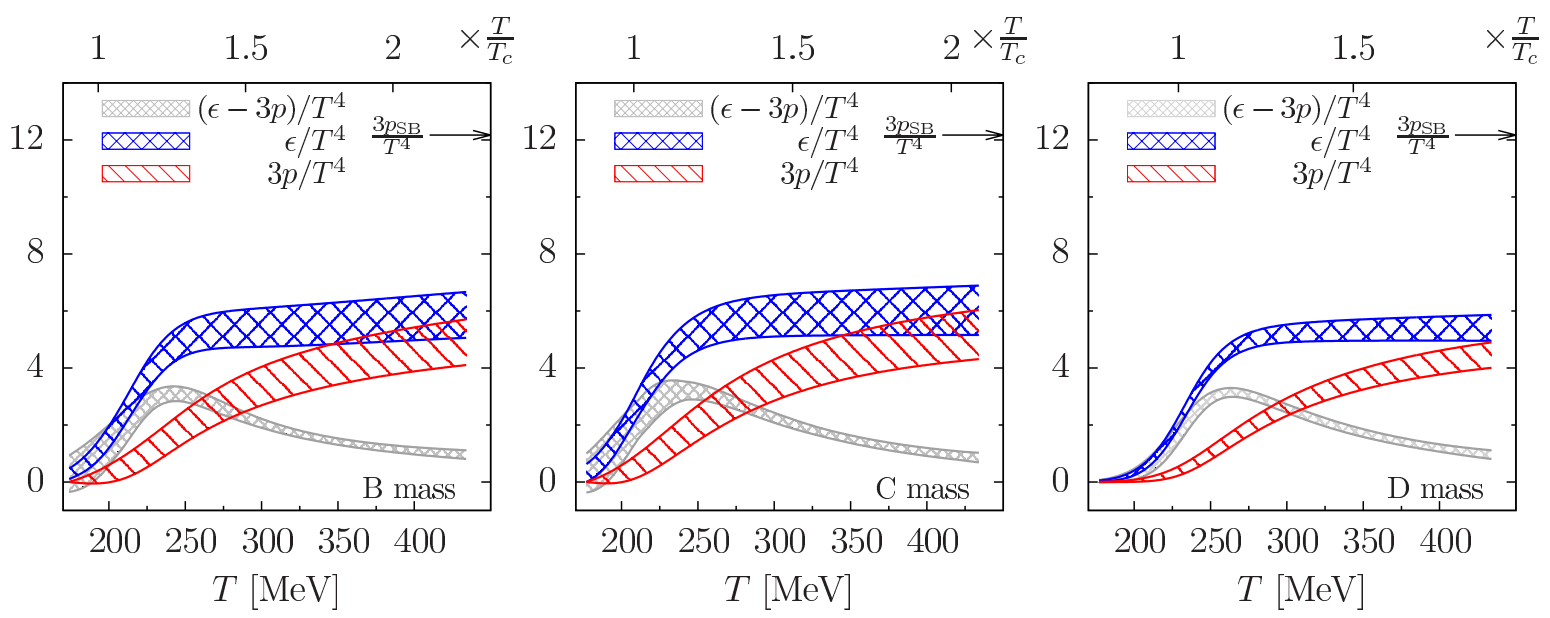

FIG. 12: Left: Final result for the pressure $p$ and the energy density $\epsilon$ in units of $T^{4}$ for the B mass ensemble. We also show once more the interpolation of the trace anomaly used for integrating the pressure. The arrow in the upper right corner indicates the expected Stefan-Boltzmann limit for the pressure. On top of the panels we provide the temperature in units of $T_{c} \equiv T_{\chi}$. Middle: The same for the C mass. Right: The same for the D mass.

In Fig. 13 we show our result for the ratio of pressure and energy density as well as the speed of sound $c_{s}^{2}$ as a function of the energy density in units of $\mathrm{GeV} / \mathrm{fm}^{3}$. The ratio $p / \epsilon$ is evaluated most directly from $p$ and $\epsilon$, whereas the speed of sound $c_{s}^{2}$ is evaluated according to Eq. 30 from its derivative. We do not calculate 

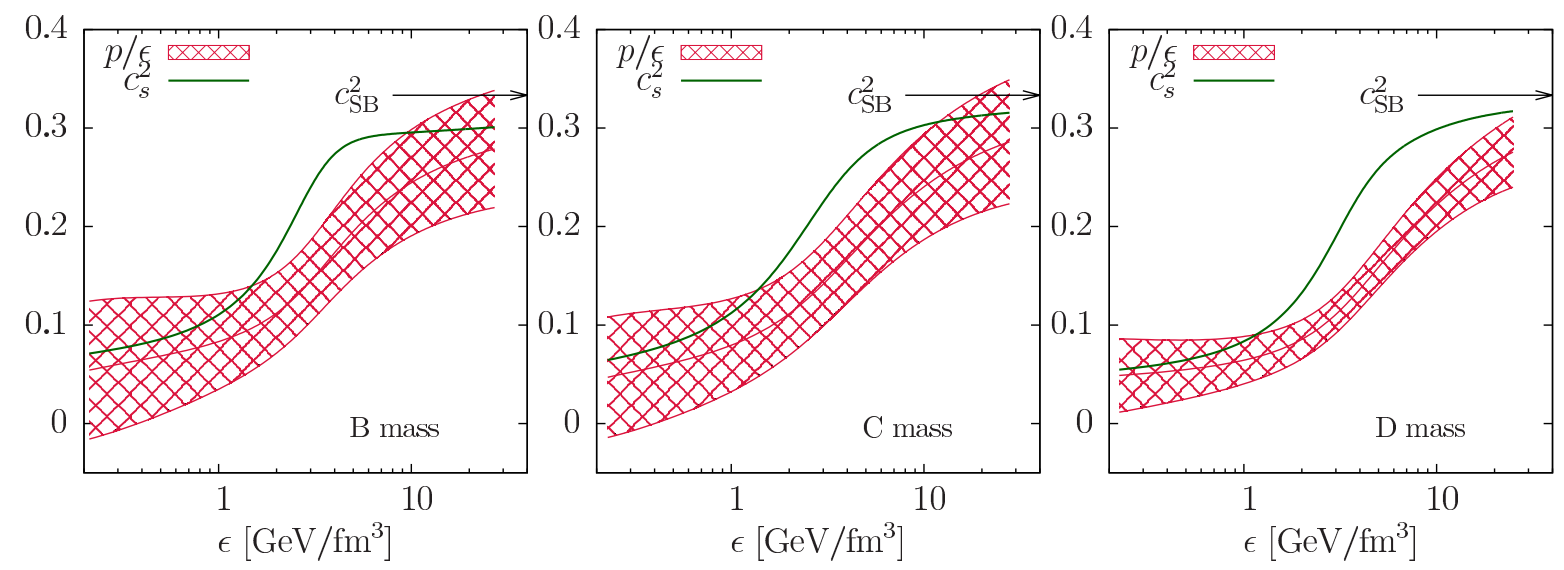

FIG. 13: Left: The ratio $p / \epsilon$ for the B ensemble as a function of the energy density in units of $\mathrm{GeV} / \mathrm{fm}^{3}$. We also show the speed of sound squared $c_{s}^{2}$ obtained from $p / \epsilon$. Arrows indicate the expected large $T$ Stefan-Boltzmann limit given by $1 / 3$. Middle: The same for the $\mathrm{C}$ mass. Right: The same for the $\mathrm{D}$ mass.

any error so far for the velocity of sound, as the error on the basic quantity $p / \epsilon$ itself is already very large. At large temperatures we observe that the limiting Stefan-Boltzmann value of $(p / \epsilon)_{\mathrm{SB}}=1 / 3$ is nicely approached. However, we are not able at the current precision to resolve the dip at small temperatures that is observed in $p / \epsilon$ results from staggered simulations [1, 37.

\section{HADRON RESONANCE GAS MODEL: FIXING THE INTEGRATION CONSTANT $p_{0}$}

In the hadronic phase at temperatures below the crossover transition hadrons and resonances form the relevant degrees of freedom that may be thermally $\mathrm{n}$ excited. It has been argued, that in this region of temperature a gas of free, non-interacting hadrons and resonances could provide a good approximation to the interacting thermal medium. A comparison of the hadron resonance gas (HRG) model with lattice data has been conducted for instance in Ref. 2, 3]. Good agreement with results of non-perturbative lattice evaluation is found for various quantities even up to the crossover temperature.

At vanishing chemical potential the free pressures of mesons $(M)$ and baryons $(B)$ can be written as

$$
\frac{p^{\mathrm{HRG}}}{T^{4}}=\frac{1}{V T^{3}} \sum_{i \in \text { Mesons }} \ln Z_{m_{i}}^{M}(T, V)+n \frac{1}{V T^{3}} \sum_{i \in \text { Baryons }} \ln Z_{m_{i}}^{B}(T, V),
$$

where according to Bose- and Fermi- statistics for mesons and baryons, respectively, and in terms of the energies $E_{i}\left(m_{i}, k\right)=\sqrt{\left(m_{i}^{2}+k^{2}\right)}$ and state degeneracies $d_{i}$ the contributions $\ln Z_{m_{i}}^{M / B}$ are given as

$$
\ln Z_{m_{i}}^{M / B}=d_{i} \frac{V}{2 \pi^{2}} \int_{0}^{\infty} k^{2} \ln \left(1 \mp e^{-E_{i}\left(m_{i}, k\right) / T}\right) d k .
$$

We have conducted a three-fold analysis confronting our lattice data of the interaction measure for the B and the $\mathrm{C}$ mass with the interaction measure provided by above formula upon taking the derivative w. r. t. temperature as prescribed in Eq. (1). There are three options. Firstly we may include all known physical states as referenced by the PDG [38] up to a certain cutoff mass, which we have set to $m_{\text {cut }}=1.9 \mathrm{GeV}$ throughout. The heaviest meson we include is the $\pi_{2}(1880)$ and the heaviest baryon is the $\Delta(1905)$. As can be seen from Fig. 14 the interaction measure evaluated with all physical states (corresponding to the $N_{f}=3$ curve in the pictures) overshoots the data at small temperatures significantly.

As another choice we may restrict the set of states entering Eq. (31) to the ones with $S=0$, i. e. to states without strangeness. Doing so already closes more than half of the gap between our lattice results and the HRG estimate from $N_{f}=2$ which is labelled " $N_{f}=2$ HRG phys." in the pictures. Since the value of pion masses we have considered in this work is yet somewhat above the physical value the remaining difference to a $N_{f}=2$ HRG model at physical masses is not unexpected. Along the ideas of Ref. [39] we have therefore conducted a third approach and have used where possible the measured lattice mass spectrum data corresponding to unphysical pion masses obtained within the ETMC. We summarize in the list given below the mass information 
$M_{B}$ and $M_{C}$ for the states we have included in this analysis for the cases of the $\mathrm{B}$ and $\mathrm{C}$ ensembles and give the reference, where it has been published:

\begin{tabular}{c|c|c|c} 
State & Reference & $M_{B}[\mathrm{GeV}]$ & $M_{C}[\mathrm{GeV}]$ \\
\hline$\rho$ & 40 & 0.943 & 0.858 \\
$a_{0}$ & $"$ & 1.116 & 1.252 \\
$b_{1}$ & $"$ & 1.603 & 1.529 \\
$\eta_{2}$ & $41]$ & 1.008 & 1.066 \\
$N$ & 42 & 1.209 & 1.282 \\
$\Delta$ & $"$ & 1.517 & 1.589
\end{tabular}

In all cases at least two lattice spacings (corresponding to $\beta=3.9$ and $\beta=4.05$ ) as well as several values of the bare quark mass $a \mu$ have been studied. A detailed continuum extrapolation including three or more values of the cutoff would go beyond our possibilities in most of the cases. For converting into physical units we have used the lattice spacings obtained in Ref. [29], $a=0.089$ and $a=0.070$ for $\beta=3.9$ and $\beta=4.05$, respectively. Since in all cases cutoff effects are small, we have used in our analysis the value obtained from a spline interpolation of the hadron mass in lattice units as a function of the bare quark mass at the finer lattice spacing and converted to physical units. The values are shown in the table above.

As the mass splitting of different isospin states induced by the twisted mass term is mostly small (with the exception of the neutral pion !) and since no cutoff effects are visible in our trace anomaly data at low temperature, we have neglected this splitting.

Furthermore, in addition to the table above we could use the $\omega-\rho$ mass-splitting of $27 \mathrm{MeV}$ calculated in Ref. [43] which then fixed the $\omega$-mass in our analysis. The excited states of the $\rho, a, b, \eta, \omega, N$ and $\Delta$ particles have been considered by taking the mass difference between the excited and the ground state particle from PDG and adding this splitting to the ground state mass measured on the lattice. Since this analysis is intended to stay on a qualitative level only, given the unknown systematics, we do not consider errors on the hadron masses either taken from PDG or from a lattice study. Masses for other particles than listed in above table have been set to their PDG values.
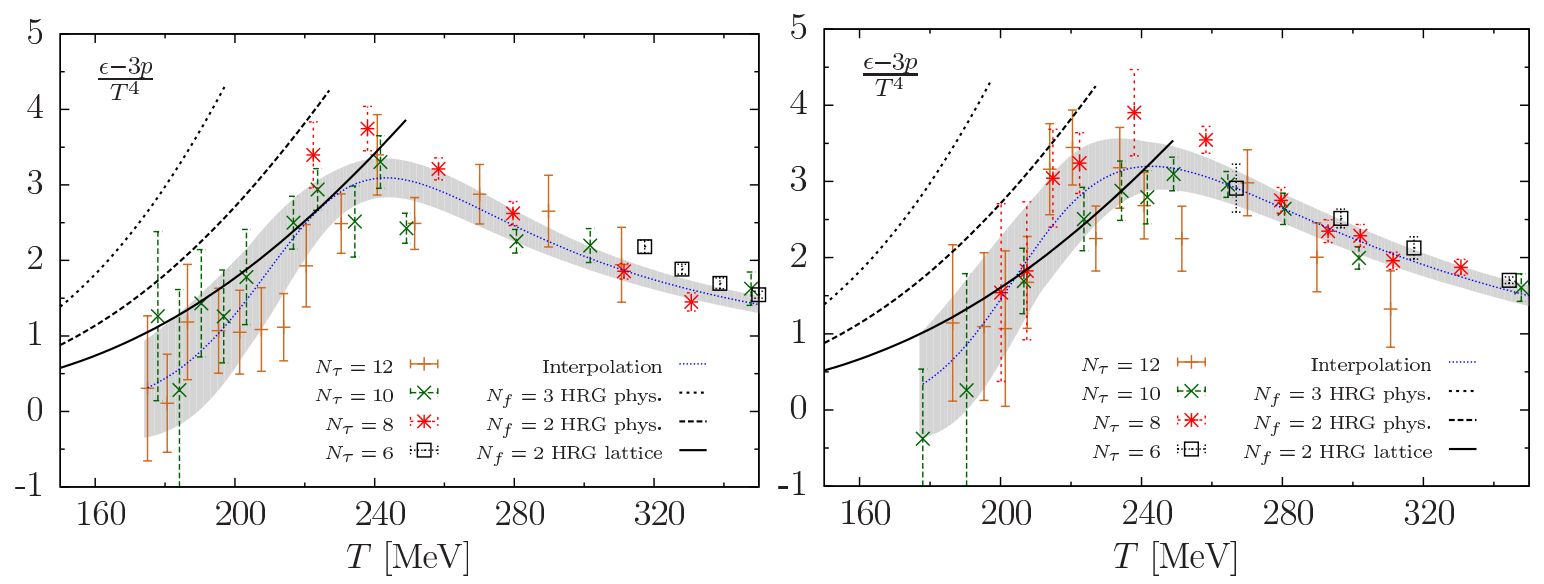

FIG. 14: Comparison of the interaction measure in the low temperature region to the predictions of several HRG model adaptations. Left: The data and analysis corresponding to the B mass is shown. Right: The same for the C mass. See text for details.

As can be seen from the curve labelled with " $N_{f}=2$ HRG lattice" in Fig. 14. this $N_{f}=2$ HRG model incorporating the unphysically high masses is compatible with our determination of the trace anomaly at temperatures in the vicinity to the transition both for the $\mathrm{B}$ and the $\mathrm{C}$ mass. In the left panel of Fig. 15 we show curves for the pressure at low temperature obtained from the various adaptations of the HRG model under consideration. The curves corresponding to the $\mathrm{B}$ and $\mathrm{C}$ mass we can use to fix the value for the integration constant $p_{0}$. At $T=174 \mathrm{MeV}$ we obtain for the $\mathrm{B}$ mass $p_{0}^{B}=0.302$ and at $T=177 \mathrm{MeV}$ we obtain for the $\mathrm{C}$ mass $p_{0}^{C}=0.267$. Using these values to start the pressure integral and assuming a conservative $20 \%$ error on $p_{0}$ in both cases we obtain for the integrated pressure and the energy density the curves depicted in the right panel of Fig. 15 . 

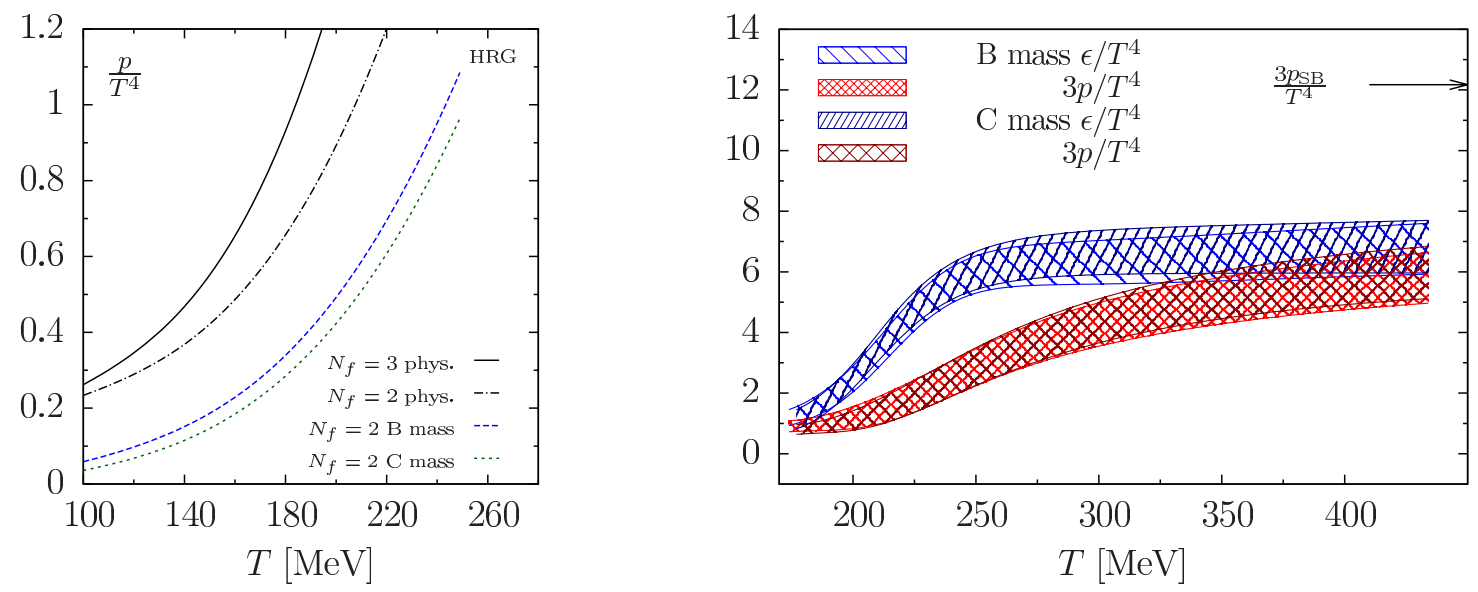

FIG. 15: Left: The pressure as obtained from several HRG models is shown. See text for details. Right: The pressure and energy density for the B mass $\left(m_{\pi} \sim 360 \mathrm{MeV}\right)$ and for the $\mathrm{C}$ mass $\left(m_{\pi} \sim 430 \mathrm{MeV}\right)$ as obtained when using the lattice HRG model pressure for estimating the integration constant $p_{0}$.

\section{COMPARISON WITH OTHER RESULTS}

Since the trace anomaly is the starting point for all bulk thermodynamics observables it is natural to choose this quantity for a comparison with other results. In our $N_{f}=2$ study the maximum of the trace anomaly has a height of $\sim 3$. Continuum extrapolated results for $N_{f}=2+1$ at the physical point are reported for stout staggered quarks in Ref. [1]) and for HISQ staggered quarks in Ref. [3. Both report the maximum of the trace anomaly at height $\sim 4$. A study using Wilson quarks together with the fixed scale approach reports the maximum at a value of $\sim 7.5[8$. We compare our result for the trace anomaly at the smallest mass with a peak height of $\sim 3$ with the data of Ref. 3 in the right panel of Fig. 16. The data is shown as a function of the ratio $T / T_{c}$, where we use our estimates from Table $\mathrm{I}$ at the largest available $N_{\tau}$ for $T_{c}$. It is also worthwile to compare with the $N_{f}=0$ case for which the EoS was computed in Ref. 44 and more recently with increased precision in Ref. 45]. The continuum extrapolated data taken from Table 1 of latter reference is also shown in Fig.16. We have connected data points with lines to guide the eye. The $N_{f}=0$ peak value is smaller than for $N_{f}=2$, and the falling edge of the trace anomaly stays below our interpolation for the B mass. The curves from our two larger quark masses seem to approach the $N_{f}=0$ curve at large temperature.

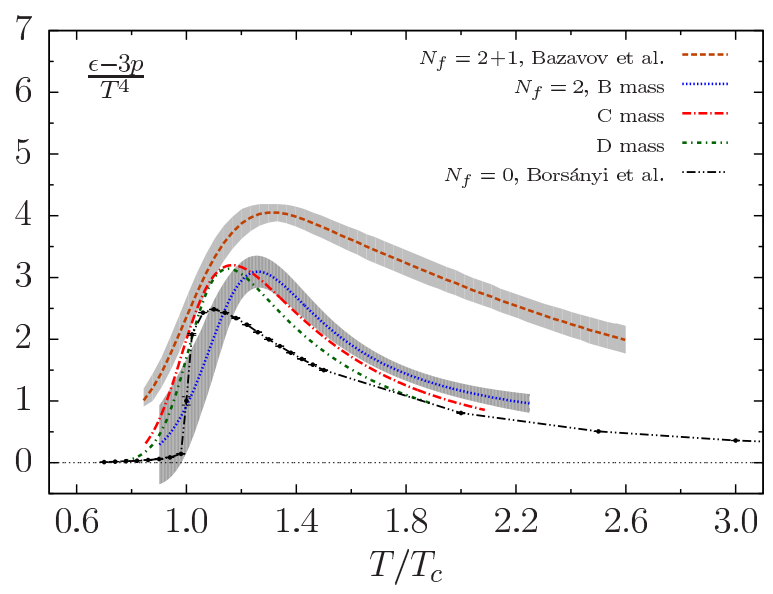

FIG. 16: A comparison of $I / T^{4}$ versus $T / T_{c}$ between $N_{f}=0$ obtained in Ref. [4], our data at $N_{f}=2$ for the B mass and $N_{f}=2+1$ obtained in Ref. 3. We also show our curves for the C and D $\left(m_{\pi} \sim 640 \mathrm{MeV}\right)$ masses, however, suppressing the errors for better visibility. For $T_{c}$ we use our $T_{\chi}$ estimates obtained at the largest $N_{\tau}$ available.

When including a non-zero $p_{0}$ as a starting point for the pressure integration we obtain at $T=2 T_{c}$ a value of $p / p_{\mathrm{SB}}=0.45(7)$ and $0.48(7)$ for the $\mathrm{B}$ and $\mathrm{C}$ mass, respectively. These values are slightly smaller than those computed with $N_{f}=2+1$ at the physical point in References [1] and [3]. 


\section{CONCLUSIONS}

In this work we have presented a calculation of QCD thermodynamics with two degenerate flavors of Wilson twisted mass quarks. To our knowledge this is the first work at $N_{f}=2$ providing a continuum limit estimate of thermodynamics. Moreover, our work constitutes the first fully systematic determination of the trace anomaly using a Wilson type quark discretization. Since we were not (yet) able to work at the physical value of the pion mass, we have conducted the calculation at three values of unphysically large pion mass. Comparing the results as a function of $T / T_{c}$ we found only little residual mass dependence for the trace anomaly, while $T_{c}$ itself decreases with smaller mass as was seen in Ref. 24]. Here we investigated the pseudo-critical temperature further at several lattice spacings for each considered (charged) pion mass and found no significant $N_{\tau}$ dependence.

The trace anomaly depends on the quark mass. For a small mass interval we find that this dependence is mostly due to a shift in $T_{c}$, since the results expressed as a function of $T / T_{c}$ show little mass dependence. However, there is a clear sensitivity to the matter content which is seen by comparing with the quenched determination and results with a dynamical strange mass, and lighter quarks. The peak height of the interaction measure is steadily increasing when enlarging the number of active quark flavors. This suggests that the QGP is more strongly interacting when adding more fermions which confirms and extends the analysis of Ref. [46] and Ref. [47.

On the basis of a continuum estimate of the trace anomaly using an interpolation ansatz we have calculated by means of the integral method (up to an integration constant) the pressure, the energy density and the speed of sound in the transition region and up to $\sim(2.0-2.5) T_{c}$.

We have compared our findings for the trace anomaly at the two lower quark mass values in the region of the transition with adaptations of the hadron resonance gas model. In order to reproduce (within errors) in these two cases of lower quark mass the rising part of the trace anomaly, not only hadrons with strangeness had to be disregarded in the model, but also the masses of their ground and also excited states had to be adapted to match the non-physical masses used in our simulations. With these adaptations to the HRG model we found it to agree with our trace anomaly results. Given this agreement we have extracted from the HRG model a value of the integration constant for the pressure at our smallest available temperatures.

\section{Acknowledgments}

F.B. and M.M.P. acknowledge support by the Corroborative Research Center SFB / Transregio 9 Computational Particle Physics. We are grateful to the HLRN supercomputing centers Berlin and Hannover for generous allocations of computation time. We thank M. Kirchner, C. Pinke, C. Urbach and L. Zeidlewicz for simulating part of the B12, C12 and B10 gauge field ensembles used in this analysis as well as O. Philipsen for fruitful discussions and hints. We are grateful to the European Twisted Mass Collaboration and its members for generating and providing $T=0$ gauge field ensembles and for continuous support. We thank M. Wagner for providing us with the code for the determination of $\left(\frac{r_{0}}{a}\right)$ as well as E. Garcia-Ramos for providing us with information about the $\beta=4.35, a \mu=0.00175$ ETMC gauge ensemble prior to publication. For the generation of gauge field configurations we have used tmLQCD [48, 49] on massively-parallel systems as well as on GPU-equipped PC-clusters. Statistical analyses were done with the help of R[50].

\section{Appendix A: Symanzik expansion of $\langle\bar{\chi} \chi\rangle_{\text {sub }}$}

Starting from Eq. 14 we need to measure a term $\left\langle\sum_{x} \bar{\chi}_{x} \chi_{x}\right\rangle_{\text {sub }}$ stemming from the $m$-derivative of the action Eq. (4). Since the sum is composed of short distance contributions and the operator may mix with different operators of the same symmetry the usual arguments of automatic $\mathcal{O}(a)$ improvement does not necessarily hold. In [51] the Symanzik expansion of the vacuum polarization tensor has been studied. We rely for the following argument on the characterization of operators in terms of symmetry transformations achieved there.

At maximal twist the symmetry transformations of $\langle\bar{\chi} \chi\rangle_{\text {sub }}$ (we suppress the sum over spacetime in what follows) read:

\begin{tabular}{c|c|c|c|c|c|c|c} 
& $\mathcal{P}_{1 / 2}$ & $\mathcal{P}_{[\mu \rightarrow-\mu]}$ & $\mathcal{T}_{1 / 2}$ & $\mathcal{T}_{[\mu \rightarrow-\mu]}$ & $\mathcal{C}$ & $\mathcal{P} \mathcal{D}[-m][-r]$ & $\mathcal{R}_{5}^{1 / 2} \mathcal{D}[-\mu]$ \\
\hline $\bar{\chi} \chi$ & +1 & +1 & +1 & +1 & +1 & -1 & +1
\end{tabular}

Being an operator of mass dimension three $\bar{\chi} \chi$ will mix with operators of mass dimension lower or equal to three under renormalization which have the same symmetry transformation properties. The local operators having 
the same symmetry properties as $\bar{\chi} \chi$ up to dimension 4 read:

$$
\left\{r, m_{q}, r m_{q}^{2}, r m_{q} \bar{\chi} \chi, r \mu_{q} \bar{\chi} \gamma_{5} \tau^{3} \chi\right\}
$$

where $m_{q}$ is to be considered as the subtracted quark mass $m_{q}=m-m_{c}$ with $m_{c}$ denoting the critical value.

Using the above set a finite subtracted operator can be constructed as:

$$
\bar{\chi} \chi_{R}=Z^{\bar{\chi} \chi} \bar{\chi} \chi+r \frac{Z^{r}}{a^{3}}+m_{q} \frac{Z^{m_{q}}}{a^{2}}+m_{q}^{2} \frac{Z^{m_{q}^{2}}}{a}+a r m_{q} Z^{r m_{q}}+a r \mu_{q} Z^{r \mu_{q}^{2}}+\mathcal{O}\left(a^{2}\right) .
$$

The expansion becomes complete once also the effective Lagrangian is expanded:

$$
\mathcal{L}_{\text {eff }}=\mathcal{L}_{4}+a \mathcal{L}_{5}+a^{2} \mathcal{L}_{6}+a^{3} \mathcal{L}_{7}+\mathcal{O}\left(a^{4}\right)
$$

From the expansion of the effective action $S$ in the Boltzmann weight

$$
\begin{aligned}
\exp (-S)=\exp (- & \left.S_{4}\right)\left\{1-a S_{5}+a^{2}\left(\frac{1}{2} S_{5}^{2}-S_{6}\right)\right. \\
+ & \left.a^{3}\left(-\frac{1}{6} S_{5}^{3}+S_{5} S_{6}-S_{7}\right)+\mathcal{O}\left(a^{4}\right)\right\}
\end{aligned}
$$

further terms arise in the Symanzik expansion of the operator, where the notation $S_{i}=\int \mathcal{L}_{i}(x) d^{4} x$ has been used. Keeping only terms up to order $\mathcal{O}(a)$ and restricting to the case $m_{q} \equiv 0$ that is implied by the maximal twist condition the combined Symanzik expansion is given by:

$$
\begin{aligned}
\langle\bar{\chi} \chi\rangle_{\mathrm{R}} & =Z^{\bar{\chi} \chi}\langle\bar{\chi} \chi\rangle_{0}+r \frac{Z^{r}}{a^{3}}\langle 1\rangle_{0}+r \frac{Z^{r}}{a^{2}}\left\langle-\mathcal{S}_{5}\right\rangle_{0} \\
& +r \frac{Z^{r}}{a}\left(\left\langle-\mathcal{S}_{6}+\frac{1}{2} S_{5}^{2}\right\rangle_{0}\right) \\
& +r Z^{r}\left(\left\langle-S_{7}+S_{5} S_{6}-\frac{1}{6} S_{5}^{3}\right\rangle_{0}\right)+\mathcal{O}(a) .
\end{aligned}
$$

The divergent terms $\sim a^{-n}$ need to be cared for by subtracting the $T=0$ result. The two finite terms $\langle\bar{\chi} \chi\rangle_{0}$ and $r Z^{r}\left\langle\mathcal{L}_{7}\right\rangle_{0}$ vanish due to $\mathcal{R}_{5}^{1 / 2}$ symmetry of $\mathcal{L}_{4}$ over which the terms are averaged, since they are $\mathcal{R}_{5}^{1 / 2}$-odd. After $T=0$ subtraction we thus note that the remaining terms are lattice artefacts of $\mathcal{O}(a)$ and higher.

For the trace anomaly we however need to consider the bare dimensionless lattice operator $a^{3}\langle\bar{\chi} \chi\rangle_{0}$ instead of the fully subtracted and multiplicatively renormalized operator discussed above. Restricting the expansion to $\mathcal{O}\left(a^{2}\right)$ precision we obtain

$$
\begin{aligned}
\frac{a^{3}}{Z \bar{\chi} \chi}\langle\bar{\chi} \chi\rangle_{\text {sub }} & =a^{3}\langle\bar{\chi} \chi\rangle_{0}+r \tilde{Z}^{r}\langle 1\rangle_{0}+a r \tilde{Z}^{r}\left\langle-\mathcal{S}_{5}\right\rangle_{0} \\
& +r a^{2} \tilde{Z}^{r}\left(\left\langle-\mathcal{S}_{6}+\frac{1}{2} S_{5}^{2}\right\rangle_{0}\right)+\mathcal{O}\left(a^{3}\right),
\end{aligned}
$$

where we have absorbed the $Z^{\bar{\chi} \chi}$ on the right hand side into the definition of the $Z$-factors of the operators. Since the bare operator $\bar{\chi} \chi$ is an $\mathcal{R}_{5}^{1 / 2}$-odd (or equally a twisted parity odd) operator its expectation value with respect to the $\mathcal{R}_{5}^{1 / 2}$-symmetric (twisted parity even) continuum twisted mass action vanishes. The constant piece proportional to $\langle 1\rangle_{0}$ will be eliminated by the $T=0$ subtraction of the trace anomaly. The remaining terms are lattice artefacts that can be further restricted to being of order $\mathcal{O}\left(a^{2}\right)$ and higher upon noting that also the contributions to $S_{5}$ are $\mathcal{R}_{5}^{1 / 2}$-odd. The $T=0$ subtracted contribution to the trace anomaly from the $m$ derivative of the action is thus seen to be vanishing in the continuum limit at $\mathcal{O}\left(a^{2}\right)$ and can (and also should) be disregarded in the evaluation right from the beginning. 


\section{Appendix B: Tables}

\begin{tabular}{|c|c|c|c|c|c|c|c|c|}
\hline$N_{\tau} N_{\sigma}$ & $\beta$ & $\kappa$ & $a \mu$ & $a m_{\mathrm{PS}}$ & $m_{\mathrm{PS}} L$ & $\left\langle S_{g}\right\rangle$ & $\left\langle S_{f}\right\rangle\left(\cdot 10^{2}\right)$ & $\left(\frac{r_{0}}{a}\right)$ \\
\hline 4824 & 3.80 & 0.164111 & 0.00600 & $0.1852(9)$ & 4.4 & $5.34639(94)$ & $4.467(16)$ & $4.321(32)$ \\
\hline 4824 & 3.80 & 0.164111 & 0.00800 & $0.2085(8)$ & 5.0 & $5.34747(60)$ & $5.590(09)$ & $4.440(34)$ \\
\hline 4824 & 3.80 & 0.164111 & 0.01100 & $0.2424(5)$ & 5.8 & $5.34900(82)$ & $7.164(07)$ & $4.362(21)$ \\
\hline 4824 & 3.80 & 0.164111 & 0.01650 & $0.2957(5)$ & 7.1 & $5.34887(22)$ & $10.001(08)$ & $4.264(14)$ \\
\hline 6432 & 3.90 & 0.160856 & 0.00300 & $0.1167(4)$ & 3.7 & - & - & - \\
\hline 6432 & 3.90 & 0.160856 & 0.00400 & $0.1338(2)$ & 4.3 & $5.47032(15)$ & $2.753(07)$ & - \\
\hline 4824 & 3.90 & 0.160856 & 0.00400 & - & - & $5.47012(33)$ & $2.737(08)$ & $5.196(28)$ \\
\hline 3216 & 3.90 & 0.160856 & 0.00400 & - & - & $5.47108(54)$ & $2.490(21)$ & . \\
\hline 4824 & 3.90 & 0.160856 & 0.00640 & $0.1694(4)$ & 4.1 & $5.47027(17)$ & $3.923(09)$ & $5.216(27)$ \\
\hline 4824 & 3.90 & 0.160856 & 0.00850 & $0.1940(5)$ & 4.7 & $5.47011(27)$ & $4.926(10)$ & $5.130(28)$ \\
\hline 4824 & 3.90 & 0.160856 & 0.01000 & $0.2100(5)$ & 5.0 & $5.47074(18)$ & $5.654(11)$ & $5.143(25)$ \\
\hline 4824 & 3.90 & 0.160856 & 0.01500 & $0.2586(7)$ & 6.2 & $5.47003(27)$ & $8.030(10)$ & $5.039(21)$ \\
\hline 6432 & 4.05 & 0.157010 & 0.00300 & $0.1038(6)$ & 3.3 & $5.63404(19)$ & $1.691(05)$ & $6.584(34)$ \\
\hline 6432 & 4.05 & 0.157010 & 0.00600 & $0.1432(6)$ & 4.6 & $5.63419(08)$ & $3.019(03)$ & $6.509(38)$ \\
\hline 6432 & 4.05 & 0.157010 & 0.00800 & $0.1651(5)$ & 5.3 & $5.63419(14)$ & $3.879(05)$ & $6.494(36)$ \\
\hline 6432 & 4.05 & 0.157010 & 0.01200 & $0.2025(8)$ & 6.5 & $5.63400(06)$ & $5.612(04)$ & $6.284(22)$ \\
\hline 9648 & 4.20 & 0.154073 & 0.00200 & $0.0740(3)$ & 3.6 & $5.78133(06)$ & $1.0013(29)$ & $8.295(45)$ \\
\hline 6432 & 4.20 & 0.154073 & 0.00650 & $0.1326(5)$ & 4.2 & $5.78130(05)$ & $2.8200(33)$ & $8.008(29)$ \\
\hline 6432 & 4.35 & 0.151740 & $\mid 0.00175$ & $0.0748(17)$ & 2.4 & $5.91477(04)$ & $0.7363(48)$ & $9.9(2)$ \\
\hline
\end{tabular}

TABLE IV: Parameters and results for $T=0$ ETMC generated gauge ensembles used in this analysis. For further details we refer the reader to Refs. [30, 31]. We show results for the pseudoscalar mass, $\left(\frac{r_{0}}{a}\right)$, the gauge action $\left\langle S_{g}\right\rangle$ and the fermion action $\left\langle S_{f}\right\rangle$. The latter two have been evaluated fully using the available statistics. 


\begin{tabular}{|c|c|c|c|c|c|c|c|c|c|c|}
\hline$N_{\tau} N_{\sigma}$ & $\beta$ & $\kappa$ & $a \mu$ & $a m_{\mathrm{PS}}$ & $m_{\mathrm{PS}} L$ & $a m_{\mathrm{PCAC}}$ & $\left\langle S_{g}\right\rangle$ & $\left\langle S_{f}\right\rangle\left(\cdot 10^{2}\right)$ & $\left(\frac{r_{0}}{a}\right)$ & $\mathrm{TU}$ \\
\hline 2416 & .65 & 0.170250 & 0.01200 & $0.302(6)$ & 4.8 & $7(9) \cdot 10^{-4}$ & - & - & $3.437(33)$ & 17396 \\
\hline 4016 & 3.65 & 0.170200 & 0.02517 & $0.425(7)$ & 6.8 & $\cdot 10^{-3}$ & - & - & $3.249(64)$ & 3610 \\
\hline 2416 & 3.70 & 0.168062 & 0.00900 & $0.254(8)$ & 4.0 & $3.6(1.0) \cdot 10^{-3}$ & $5.2231(12)$ & $7.356(29)$ & $3.853(59)$ & 29042 \\
\hline 2416 & 3.70 & 0.168062 & 0.01055 & $0.268(6)$ & 4.3 & $-2.6(7) \cdot 10^{-3}$ & $5.2212(13)$ & $8.331(23)$ & $3.706(116)$ & 15126 \\
\hline 4020 & 3.70 & 0.168092 & 0.02406 & $0.397(7)$ & 7.9 & $-4.8(9) \cdot 10^{-3}$ & $5.21675(32)$ & $16.085(16)$ & $3.673(40)$ & 7359 \\
\hline 2020 & 3.72 & 0.167216 & 0.00724 & - & - & - & - & - & $3.895(51)$ & 5637 \\
\hline 2020 & 3.72 & 0.167229 & 0.02342 & - & - & - & $5.24341(52)$ & $15.236(22)$ & $3.721(38)$ & 3017 \\
\hline 2020 & 3.74 & 0.166401 & 0.02279 & - & - & - & $5.26912(46)$ & $14.396(15)$ & - & 6237 \\
\hline 2416 & 3.76 & 0.165607 & 0.00689 & $0.208(5)$ & 3.3 & $5(6) \cdot 10^{-4}$ & $5.29840(53)$ & $5.291(19)$ & $4.186(52)$ & 52665 \\
\hline 2416 & 3.76 & 0.165607 & 0.00979 & $0.246(6)$ & 3.9 & $-1.1(7) \cdot 10^{-3}$ & $5.29826(54)$ & $6.979(10)$ & $4.058(97)$ & 20000 \\
\hline 4020 & 3.76 & 0.165608 & 0.02218 & $0.354(9)$ & 7.0 & $-2.1(9) \cdot 10^{-3}$ & $5.29581(24)$ & $13.652(08)$ & $3.972(24)$ & 7405 \\
\hline 2020 & 3.78 & 0.164844 & 0.02158 & - & - & - & $5.32171(49)$ & $12.916(15)$ & - & 2900 \\
\hline 3220 & 3.80 & 0.164111 & 0.00655 & - & & - & $5.34846(27)$ & $4.787(08)$ & - & 24343 \\
\hline 4020 & 3.80 & 0.164111 & 0.02100 & $0.338(4)$ & 6.7 & $-1.8(9)$ & $5.34767(28)$ & $12.274(11)$ & $4.166(51)$ & 5319 \\
\hline 4824 & 3.82 & 0.163407 & 0.00639 & - & - & - & $5.37469(76)$ & $4.538(12)$ & - & 2263 \\
\hline 2020 & 3.82 & 0.163407 & 02043 & - & - & - & $.37207(49)$ & 11.672 & - & 3030 \\
\hline 4824 & 3.84 & 0.162731 & 0.00623 & - & - & - & 991 & 4.281 & - & 1941 \\
\hline 2020 & 3.84 & 0.162731 & 0.01989 & - & - & - & $5.39851(37)$ & $11.066(19)$ & - & 2541 \\
\hline 4824 & 3.85 & 0.162403 & 0.00600 & $0.175(2)$ & 4.2 & $-1(4) \cdot 10$ & & & 3) & 1244 \\
\hline 4824 & 3.85 & 0.162403 & 0.00893 & $0.208(4)$ & 5.0 & $0.2(4.4) \cdot 10^{-4}$ & $5.41094(17)$ & $5.573(06)$ & $4.684(43)$ & 4881 \\
\hline 4020 & 3.85 & 0.162403 & 0.01962 & $0.311(6)$ & 6.2 & $\cdot 10^{-3}$ & 5.41025 & $10.799(11)$ & $4.550(52)$ & 4880 \\
\hline 4824 & 3.86 & 0.162081 & 0.00617 & $0.174(2)$ & 4.1 & $-3(3) \cdot 10^{-4}$ & $5.42323(21)$ & $4.092(06)$ & - & 10054 \\
\hline 2020 & 3.86 & 0.162081 & 0.01935 & - & - & - & $5.42195(29)$ & 10.553 & - & 3003 \\
\hline 2020 & 3.87 & 0.161766 & 0.01909 & - & - & - & $5.43396(20)$ & 10.285 & - & 4517 \\
\hline 4824 & 3.88 & 0.161457 & 0.00600 & $0.168(5)$ & 4.0 & $-7(4) \cdot 10^{-4}$ & $5.44729(19)$ & 3.857( & - & 9528 \\
\hline 2020 & 3.88 & 57 & 83 & - & - & - & $5.44576(24)$ & $10.060(19)$ & - & 4032 \\
\hline 4020 & 3.90 & 0.160856 & 0.01833 & $0.292(6)$ & 5.8 & $-1.9(9) \cdot 10^{-3}$ & $5.46948(18)$ & $9.589(09)$ & $4.842(44)$ & 2522 \\
\hline 2020 & 3.92 & 0.160278 & 0.01784 & - & - & - & $5.49305(23)$ & $9.111(12)$ & - & 2899 \\
\hline 4824 & 3.93 & 998 & 0.00561 & $0.158(3)$ & 3.7 & $-1(3) \cdot 10^{-4}$ & $5.50477(22)$ & $3.345(04)$ & $5.447(61)$ & 9324 \\
\hline 4824 & 3.93 & 998 & 0.00801 & $0.182(8)$ & 4.4 & $-8(7) \cdot 10^{-3}$ & $5.50495(24)$ & $4.486(08)$ & $5.367(72)$ & 2817 \\
\hline 2020 & 3.93 & 997 & 0.01759 & - & - & - & $5.50434(18)$ & $8.917(25)$ & $5.324(83)$ & 4437 \\
\hline 2020 & 3.94 & 0.159722 & 0.01736 & - & - & - & $5.51565(25)$ & $8.717(13)$ & - & 2809 \\
\hline 4824 & 3.95 & 0.159452 & 0.00546 & $0.151(3)$ & 3.6 & $2(2) \cdot 10^{-4}$ & $5.52714(11)$ & 3.185 & - & 6955 \\
\hline 2020 & 3.96 & 59187 & 0.01689 & - & - & - & $5.53763(21)$ & & - & 2045 \\
\hline 6432 & $|3.97|$ & 27 & 0.00531 & $0.144(1)$ & 4.6 & $\cdot 10^{-4}$ & 5.5494 & $3.013(06)$ & $5.809(112)$ & 2299 \\
\hline 4824 & 3.97 & 0.158926 & 0.00752 & $0.176(7)$ & 4.2 & $-6(7) \cdot 10^{-4}$ & $5.54901(10)$ & $4.036(04)$ & $5.733(59)$ & 4800 \\
\hline 4020 & 3.97 & 0.158926 & 0.01666 & $0.263(4)$ & 5.2 & $-1.7(8) \cdot 10^{-3}$ & $880(12)$ & $151(10)$ & $455(55)$ & 4211 \\
\hline 2020 & 3.98 & 571 & 0.01644 & . & - & - & $5.55936(21)$ & $7.972(10)$ & - & 2806 \\
\hline 4824 & 3.99 & 0.158421 & 0.00517 & $0.141(4)$ & 3.3 & $-2(3) \cdot 10^{-4}$ & $5.57097(11)$ & $2.856(05)$ & - & 5538 \\
\hline 6432 & 4.01 & 0.157933 & 0.00503 & $0.135(2)$ & 4.3 & $-3(3) \cdot 10^{-4}$ & $5.59268(16)$ & $2.722(10)$ & - & 926 \\
\hline 4824 & 4.01 & 0.157933 & 0.00718 & $0.164(4)$ & 3.9 & $-3(4) \cdot 10^{-4}$ & $5.59207(10)$ & $3.690(05)$ & - & 5264 \\
\hline 4020 & 4.05 & 0.157010 & 0.01520 & $0.233(8)$ & 4.6 & $-3(2) \cdot 10^{-3}$ & $5.63380(09)$ & $6.974(09)$ & $6.233(76)$ & 4180 \\
\hline 6432 & 4.10 & 0.155945 & 0.00445 & $0.117(2)$ & 3.7 & $2(1) \cdot 10^{-4}$ & $5.68485(06)$ & $2.192(05)$ & - & 2090 \\
\hline 2020 & 4.10 & 0.155946 & 0.01431 & - & - & - & $5.68453(18)$ & $6.334(03)$ & - & 1485 \\
\hline 4824 & 4.20 & 0.154073 & 0.01000 & $0.16(2)$ & 3.8 & $4(12) \cdot 10^{-4}$ & $5.78135(11)$ & $4.205(08)$ & $7.6(2)$ & 810 \\
\hline 4020 & 4.20 & 0.154073 & 0.01270 & $0.20(2)$ & 4.0 & $-3(2) \cdot 10^{-3}$ & $5.78114(08)$ & $5.254(08)$ & - & 3432 \\
\hline 4824 & 4.35 & 0.151740 & 0.00600 & $0.14(3)$ & 3.3 & $-8(20) \cdot 10^{-4}$ & $5.91479(09)$ & $2.363(06)$ & $9.61(49)$ & 977 \\
\hline 4020 & 4.35 & $0.151740 \mid$ & $0.01050 \mid$ & $|0.176(8)|$ & 3.5 & $-0.2(9) \cdot 10^{-3}$ & $5.91485(08)$ & $4.069(18)$ & $10.19(55)$ & 3907 \\
\hline
\end{tabular}

TABLE V: Simulation parameters for the $T=0$ runs. We show results for the pseudoscalar and PCAC mass as well as $\left(\frac{r_{0}}{a}\right)$ where calculated. 


\begin{tabular}{|c|c|c|c|c|c|c|c|c|c|}
\hline$N_{\tau}$ & $N_{\sigma}$ & $p$ & $\kappa$ & $a \mu$ & $T[\mathrm{MeV}]$ & $\operatorname{Re}(L)$ & $\left\langle S_{g}\right\rangle$ & $\left\langle S_{f}\right\rangle\left(\cdot 10^{2}\right)$ & $\mathrm{TU}$ \\
\hline \multirow[t]{15}{*}{12} & 32 & 3.86 & 0.162081 & 0.00617 & 175 & $5.9(3) \cdot 10^{-4}$ & $5.42332(22)$ & $3.937(08)$ & 1669 \\
\hline & & 88 & 0.161457 & 00600 & 181 & $7.3(3) \cdot 10^{-4}$ & $5.44716(13)$ & $3.691(06)$ & 17375 \\
\hline & & 3.90 & 0.160856 & 0.00584 & 186 & $.7(2) \cdot 10^{-4}$ & $5.470^{7}$ & $3.440(07)$ & 4249 \\
\hline & & 3.93 & 0.159998 & 0.00561 & 195 & $1.24(3) \cdot 10^{-3}$ & $5.50501(13)$ & $3.114(11)$ & 12099 \\
\hline & & 3.95 & 0.159452 & 16 & 201 & & $5.52741(12)$ & 2.930 & 7878 \\
\hline & & 3.97 & 0.158927 & 0.00531 & 208 & & $5.54952(12)$ & $2.724(10)$ & 9653 \\
\hline & & 3.99 & 0.158421 & 00517 & 214 & $2.20(4) \cdot 10^{-3}$ & 5.571 & $2.557(13)$ & 8968 \\
\hline & & 4.01 & 0.157933 & 0.00503 & 220 & & 06) & 2.39 & 15223 \\
\hline & & 4.04 & 0.157235 & 0.00689 & 230 & $3.39(5) \cdot 10^{-3}$ & $5.62441(08)$ & $2.186(07)$ & 6080 \\
\hline & & 4.07 & 0.156573 & 0.00463 & 241 & & 5.655 & 2.030 & 3359 \\
\hline & & 4.10 & 0.155945 & 0.00445 & 251 & $4.84(5) \cdot 10^{-3}$ & $5.68538(06)$ & $1.894(04)$ & 15073 \\
\hline & & 4.15 & 0.154969 & 00422 & 270 & & 5.734 & 1.736 & 4080 \\
\hline & & 4.20 & 0.154073 & 0.00396 & 290 & $7.57(8) \cdot 10^{-3}$ & 5.781 & 1.582 & 4640 \\
\hline & & 4.25 & 247 & 0.00372 & 311 & & 5.8276 & 1.4510 & 4160 \\
\hline & & 4.35 & 40 & 6 & 6 & 1.2 & & & 4334 \\
\hline \multirow[t]{15}{*}{10} & 32 & 3.76 & 0.16 & 0 & 178 & $1.53(3) \cdot 10^{-3}$ & 5.298 & 5.138 & 18438 \\
\hline & & 3.78 & 0.16 & 00672 & 184 & & 5.323 & 4.756 & 10385 \\
\hline & & 3.80 & 0.164111 & 0.006 & 190 & 4) $\cdot 10^{-3}$ & 5.3497 & 4.40 & 11692 \\
\hline & & 3.82 & 407 & 0 & 1 & & 5.374 & 4.0 & 7811 \\
\hline & & 3.84 & 0.16 & 0 . & & & & $3.7^{\prime}$ & 9433 \\
\hline & & 3.88 & 457 & 0 & 217 & -3 & 5.448 & 3.2 & 7945 \\
\hline & & 3.90 & 56 & & 224 & -3 & & & 2987 \\
\hline & & 3.93 & 998 & 0 & 234 & $\cdot 10^{-3}$ & 5.506 & 2.9 & 4025 \\
\hline & & 3.95 & 0.15 & 0.00545 & 242 & $\cdot 10^{-3}$ & $5.52882(17)$ & 2.578 & 1971 \\
\hline & & 3.97 & 0.1 & 0 & 249 & $\cdot 10^{-3}$ & 5.5504 & 2.469( & 7276 \\
\hline & & 4.01 & 33 & 0 & 26 & & . & 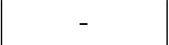 & 2720 \\
\hline & & 4.05 & 0.1 & 0 & 281 & & 5.635 & 2.060 & 8716 \\
\hline & & 4.10 & 45 & 0 & 302 & $\cdot 10^{-2}$ & 5.685 & 1.873 & 2211 \\
\hline & & 4.20 & & & & & & & 000 \\
\hline & & 4.35 & 740 & 0.003 & 428 & $2.86(2) \cdot 10^{-2}$ & 5.9 & 1.2 & 2235 \\
\hline \multirow[t]{10}{*}{8} & 28 & 3.76 & 07 & 006 & 2 & $\cdot 10^{-2}$ & 2) & 4. & 4350 \\
\hline & & 3.80 & & & & -2 & & & 4500 \\
\hline & & 3.85 & 01 & & 2 & 2) $\cdot 10^{-2}$ & 5.4 & 3.0 & 4444 \\
\hline & & 3.90 & 0.1 & 0.0 & 280 & $2.46(2) \cdot 10^{-2}$ & 5.4 & 2.72 & 3007 \\
\hline & & 3.97 & 34 & 9 & & $\cdot 10^{-2}$ & & & 3148 \\
\hline & & 4.01 & 55 & 0 & 1 & & 2) & 2.1 & 2746 \\
\hline & & 4.05 & & & & $3.80(2) \cdot 10^{-2}$ & $5.63594(11)$ & 2.011 & 3792 \\
\hline & & 4.10 & 0.1 & 0.00449 & 377 & $2) \cdot 10^{-2}$ & $5.68652(10)$ & 1.837 & 3581 \\
\hline & & 4.20 & 0.1 & 0.0 & 43 & $5.12(2) \cdot 10^{-2}$ & 5.782 & 03) & 3750 \\
\hline & & 4.35 & 0.15 & 0.00 & & $6.56(2) \cdot 10^{-2}$ & 5.91 & 1.16 & 4200 \\
\hline \multirow[t]{9}{*}{6} & 32 & 3.80 & 1 & 5 & 317 & & 7) & ) & 2926 \\
\hline & & 3.82 & & & & $7.38(3) \cdot 10^{-2}$ & $5.38576(20)$ & 3.075 & 2085 \\
\hline & & 3.84 & 0.1 & 0 & 3 & $2) \cdot 10^{-2}$ & $5.40928(22)$ & $2.9446(07)$ & 1578 \\
\hline & & 3.86 & 0.162080 & 0.0 & 350 & $7.98(2) \cdot 10^{-2}$ & $5.43233(21)$ & 2.824 & 1611 \\
\hline & & 3.90 & 0.16 & 0.00 & & $8.60(2) \cdot 10^{-2}$ & $5.47760(16)$ & $2.6033(05)$ & 2337 \\
\hline & & 3.97 & & 0. & 415 & $9.62(2) \cdot 10^{-2}$ & $5.55432(15)$ & 2.273 & 2034 \\
\hline & & 4.05 & 0.157010 & 0.004 & 468 & $1.076(2) \cdot 10^{-1}$ & $5.63805(16)$ & 1.963 & 2151 \\
\hline & & 4.10 & & & & $\cdot 10^{-1}$ & $5.68829(20)$ & $1.7979(02)$ & 1025 \\
\hline & & 4.20 & 073 & 0.00 & 580 & $1.284(1) \cdot 10^{-1}$ & $5.78360(06)$ & $1.5174(01)$ & 7440 \\
\hline \multirow[t]{4}{*}{4} & 32 & 3.80 & 0.164111 & 0.006 & 47 & $2.507(1) \cdot 10^{-1}$ & $5.38475(23)$ & $2.9518(03)$ & 2528 \\
\hline & & 3.86 & 0.1 & 0.0 & 5 & $2.605(1) \cdot 10^{-1}$ & $5.45140(20)$ & $2.6245(02)$ & 2233 \\
\hline & & $3.90 \mid$ & 0.160856 & 0.005 & 5 & $2.669(1) \cdot 10^{-1}$ & $5.49520(32)$ & $2.4324(02)$ & 1737 \\
\hline & & 3.97 & $|0.158934|$ & 0.00529 & 623 & $2.782(1) \cdot 10^{-1}$ & $5.56926(18)$ & $2.1392(02)$ & 1547 \\
\hline
\end{tabular}

TABLE VI: Simulation parameters for the B mass ensembles. Results for the bare Polyakov loop, the gauge action $\left\langle S_{g}\right\rangle$ and the fermion action abbreviated as $\left\langle S_{f}\right\rangle$. TU denotes the number of Monte Carlo time units simulated. 


\begin{tabular}{|c|c|c|c|c|c|c|c|c|c|}
\hline$N_{\tau}$ & $N_{\sigma}$ & $\underline{\beta}$ & $\kappa$ & $a \mu$ & $T[\mathrm{MeV}]$ & $\operatorname{Re}(L)$ & $\left\langle S_{g}\right\rangle$ & $\left\langle S_{f}\right\rangle$ & $\mathrm{U}$ \\
\hline \multirow[t]{14}{*}{ 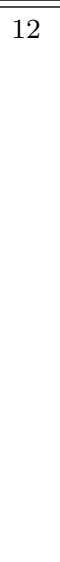 } & 32 & 0 & 856 & 21 & 186 & $8.4(5) \cdot 10^{-4}$ & $47062(16)$ & 4.65 & . \\
\hline & & 93 & 59997 & 801 & 195 & $1.16(4) \cdot 10^{-3}$ & $5.50490(17)$ & $4.309(13)$ & 180 \\
\hline & & 3.95 & 0.159452 & 0.00779 & 201 & $1.35(3) \cdot 10^{-3}$ & $5.52722(20)$ & $4.066(21)$ & 822 \\
\hline & & 3.97 & 0.158926 & 00752 & 208 & $1.63(3) \cdot 10^{-3}$ & $5.54938(13)$ & 3.818 & 179 \\
\hline & & 3.99 & 0.158421 & 0.00738 & 214 & $.13(5) \cdot 10^{-3}$ & $5.57143(10)$ & $3.603(12)$ & 5151 \\
\hline & & 4.01 & 0.157933 & 0.00718 & 220 & $2.48(5) \cdot 10^{-3}$ & $5.59288(10)$ & $3.411(16)$ & 3270 \\
\hline & & 4.03 & 0.157463 & 0.00699 & 227 & $2.92(7) \cdot 10^{-3}$ & $5.61370(09)$ & $3.241(25)$ & 6428 \\
\hline & & 4.05 & 0.157010 & 0.00680 & 234 & $3.57(8) \cdot 10^{-3}$ & 5.63471(09) & $3.063(13)$ & 2620 \\
\hline & & 4.07 & 0.156573 & 00662 & 241 & & 5.6551 & 2.905 & 3916 \\
\hline & & 4.10 & 0.155946 & 0.00639 & 251 & $4.92(7) \cdot 10^{-3}$ & $5.68525(09)$ & $2.72 ?$ & 2613 \\
\hline & & 4.15 & 0.154975 & 00599 & 270 & $6.2(1) \cdot 10^{-3}$ & 5.734 & 2.46 & 2653 \\
\hline & & 4.20 & 1073 & 3 & 290 & $7.4(2) \cdot 10^{-3}$ & & 2.24 & 2627 \\
\hline & & 4.25 & 0.153238 & 00528 & 310 & $8.6(2) \cdot 10^{-3}$ & 5.827 & 2.0612( & 2807 \\
\hline & & 4.35 & 0.151740 & 66 & 356 & $1.22(2) \cdot 10^{-2}$ & 5.915 & 1.74 & 2718 \\
\hline \multirow[t]{12}{*}{10} & 32 & 3.76 & 0.16 & 9 & 178 & $1.29(3) \cdot 10^{-3}$ & 5.297 & 6.8 & 10357 \\
\hline & & 3.80 & 111 & 6 & & & 5.349 & 6.1 & 9002 \\
\hline & & 3.85 & 0.16 & 893 & 207 & 6) $\cdot 10^{-3}$ & 5.4117 & 14) & 7679 \\
\hline & & 3.90 & 0.160856 & 00821 & 224 & $\cdot 10^{-3}$ & 5.471 & $4.267(16)$ & 10065 \\
\hline & & 3.93 & & & & & & & 7173 \\
\hline & & 3.95 & 452 & 0.00779 & 242 & $7.62(8) \cdot 10^{-3}$ & 5.528 & 3.72 & 8530 \\
\hline & & 3.97 & 0.158926 & 00752 & 249 & $\cdot 10^{-3}$ & 5.5504 & $3.497(09)$ & 6518 \\
\hline & & 4.01 & $0.1:$ & 0.00718 & 265 & 1) $\cdot 10^{-2}$ & 5.593 & $(03)$ & 11240 \\
\hline & & 4.05 & 0.15 & 00680 & 281 & & 5.635 & 2.9 & 7264 \\
\hline & & 4.10 & 0.15 & 0639 & 302 & & $5.685^{\prime}$ & 2.6 & 6864 \\
\hline & & 4.20 & 073 & 563 & 348 & & 5.781 & 2.2 & 5231 \\
\hline & & 4.35 & & & & & & 1.7331 & 5051 \\
\hline \multirow[t]{14}{*}{8} & 28 & .65 & 250 & 01200 & & 7) $\cdot 10^{-3}$ & - & & 4100 \\
\hline & & 3.70 & 0.168062 & 0.01055 & 200 & $2) \cdot 10^{-3}$ & $5.1385(13)$ & $7.589(25)$ & 4315 \\
\hline & & 3.72 & 0.16 & 1029 & 207 & 4) $\cdot 10^{-3}$ & $5.22238(91)$ & $6.987(28)$ & 4943 \\
\hline & & 3.74 & 0.16 & 4 & 5 & $\cdot 10^{-3}$ & 5.248 & 6.4 & 5199 \\
\hline & & 3.76 & 0.1 & 0979 & 222 & $1.16(2) \cdot 10^{-2}$ & $5.30285(45)$ & 5.910 & 4763 \\
\hline & & 3.80 & 11 & 00956 & & 2) $10^{-2}$ & 5.354 & 5.2 & 4745 \\
\hline & & 3.85 & & & & & & & 5130 \\
\hline & & 3.90 & 0.1 & .00821 & & $2.44(2) \cdot 10^{-2}$ & 14) & 3.8 & 4199 \\
\hline & & 3.93 & 0.15 & 0.00801 & 293 & $\cdot 10^{-2}$ & 5.5077 & 04) & 4468 \\
\hline & & 3.95 & $0.1:$ & & & $2.92(2) \cdot 10^{-2}$ & $5.53003(15)$ & 3.508 & 2640 \\
\hline & & 3.97 & 0.1 & 0.00752 & 311 & $\cdot 10^{-2}$ & 5.5515 & 3.338 & 2688 \\
\hline & & 4.01 & & 8 & $3:$ & $3.43(2) \cdot 10^{-2}$ & 5.594 & 3.0 & 4200 \\
\hline & & 4.10 & $0.1:$ & 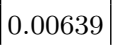 & & $4.23(3) \cdot 10^{-2}$ & $5.68634(14)$ & 2.612 & 2122 \\
\hline & & 4.20 & $0.1:$ & 563 & 435 & $5.16(2) \cdot 10^{-2}$ & 5.782 & $2.1970(04)$ & 2689 \\
\hline \multirow[t]{10}{*}{6} & 32 & 3.70 & & & & & & & 6314 \\
\hline & & 3.76 & 0.16 & 0.00979 & 2 & $6.50(2) \cdot 10^{-2}$ & $5.31365(14)$ & $5.0121(17)$ & 5351 \\
\hline & & 3.80 & 0.164111 & 0956 & 317 & $7.09(2) \cdot 10^{-2}$ & $5.36179(13)$ & $4.6898(14)$ & 4353 \\
\hline & & 3.85 & 0.16 & & & $7.85(2) \cdot 10^{-2}$ & $5.42080(11)$ & 4.183 & 4276 \\
\hline & & 3.90 & 0.16 & 0.0 & 37 & $8.60(1) \cdot 10^{-2}$ & $5.47772(10)$ & 3.69 & 6027 \\
\hline & & 3.95 & 0.159452 & 0.00779 & 403 & $9.32(2) \cdot 10^{-2}$ & $5.53282(11)$ & $3.3895(03)$ & 4520 \\
\hline & & 4.01 & & & & $(2) \cdot 10^{-1}$ & $5.59667(10)$ & $3.0112(03)$ & 4054 \\
\hline & & 4.05 & 0.157010 & 0.006 & 468 & $1.078(2) \cdot 10^{-1}$ & $5.63790(10)$ & 2.790 & 3069 \\
\hline & & 4.10 & $0.1:$ & 0.00 & 50 & $1.146(2) \cdot 10^{-1}$ & $5.68786(08)$ & $2.5554(02)$ & 5168 \\
\hline & & 4.20 & 0.154073 & 0.00 & 580 & $1.285(2) \cdot 10^{-1}$ & $5.78372(10)$ & $2.1564(02)$ & 3167 \\
\hline \multirow[t]{4}{*}{4} & 32 & 3.70 & 0.16 & 0 & 40 & $2.346(1) \cdot 10^{-1}$ & $5.27035(14)$ & $5.1616(06)$ & 4353 \\
\hline & & 3.76 & 0.165607 & 0.00979 & 445 & $2.442(1) \cdot 10^{-1}$ & $5.33934(14)$ & $4.5500(03)$ & 4840 \\
\hline & & 3.80 & 0.164 & 0.0 & 47 & $2.506(1) \cdot 10^{-1}$ & $5.38473(12)$ & $4.3062(02)$ & 4714 \\
\hline & & 3.85 & 0.162403 & 0.00893 & 517 & $2.587(1) \cdot 10^{-1}$ & $5.44025(12)$ & $3.8816(03)$ & 3463 \\
\hline
\end{tabular}

TABLE VII: Simulation parameters for the C mass ensembles. 


\begin{tabular}{|c|c|c|c|c|c|c|c|c|c|}
\hline$N$ & $N_{\sigma}$ & $\beta$ & $\kappa$ & $a \mu$ & $T[\mathrm{MeV}]$ & $\operatorname{Re}(L)$ & $\left\langle S_{g}\right\rangle$ & $\left\langle S_{f}\right\rangle(\cdot 1$ & $\mathrm{U}$ \\
\hline \multirow[t]{13}{*}{10} & 24 & 3.76 & 0.165608 & 0.02218 & 178 & $8.5(4) \cdot 10^{-4}$ & $5.29599(39)$ & $13.561(09)$ & 9076 \\
\hline & & .80 & 0.164111 & 0.02100 & 190 & $1.21(4) \cdot 10^{-3}$ & $5.34724(24)$ & $12.185(08)$ & 9391 \\
\hline & & 3.85 & 0.162401 & 0.01962 & 207 & & $5.41016(18)$ & $10.652(11)$ & 9044 \\
\hline & & 3.90 & 0.160856 & 0.01833 & 224 & $3.64(10) \cdot 10^{-3}$ & $5.47014(18)$ & $9.3163(12)$ & 9648 \\
\hline & & 3.93 & 0.159997 & 0.01759 & 234 & $4.98(15) \cdot 10^{-3}$ & $5.50473(16)$ & $8.6074(17)$ & 7742 \\
\hline & & 3.97 & 0.158934 & 0.01666 & 249 & $7.25(13) \cdot 10^{-3}$ & $5.54965(10)$ & $7.7486(11)$ & 11627 \\
\hline & & 3.99 & 158421 & 01621 & 257 & $8.47(16) \cdot 10^{-3}$ & $5.57166(12)$ & $7.3759(10)$ & 8546 \\
\hline & & 4.01 & 933 & 0.01578 & 265 & $41(24) \cdot 10^{-3}$ & 4) & $7.0487(21)$ & 4059 \\
\hline & & 4.05 & 0.157010 & 0.01524 & 281 & $1.22(2) \cdot 10^{-2}$ & $5.63506(11)$ & $6.5485(05)$ & 7210 \\
\hline & & 4.10 & 0.155946 & 01431 & 302 & & $5.68574(13)$ & 5.9618 & 3373 \\
\hline & & 4.20 & 0.154073 & 0.01261 & 348 & $2.03(2) \cdot 10^{-2}$ & $5.78192(08)$ & $4.9791(02)$ & 7059 \\
\hline & & 4.25 & 0.153241 & 01184 & 372 & $2.30(3) \cdot 10^{-2}$ & $5.82768(12)$ & $4.5758(02)$ & 3377 \\
\hline & & 4.35 & 0.151740 & 0.01043 & 428 & $2.87(3) \cdot 10^{-2}$ & 5.915 & 3.8838 & 3368 \\
\hline \multirow[t]{23}{*}{8} & 20 & 3.65 & 0.17 & 17 & 18 & 4) $\cdot 10^{-3}$ & 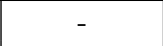 & - & 21656 \\
\hline & & 3.70 & 63 & 02406 & & & $5.21663(41)$ & $15.762(14)$ & 8240 \\
\hline & & 3.72 & 0.167219 & 0.02342 & 207 & $5.29(9) \cdot 10^{-3}$ & $5.24389(37)$ & $14.827(15)$ & 16961 \\
\hline & & 3.74 & 0.166400 & 0.02279 & 215 & $8) \cdot 10^{-3}$ & $5.27051(29)$ & $13.942(14)$ & 24771 \\
\hline & & 3.76 & 0.16 & 0.02218 & 222 & $8.1(2) \cdot 10^{-3}$ & 5.2975 & $13.104(22)$ & 18392 \\
\hline & & 3.78 & 0.16 & 0.02158 & 230 & & 5.3242 & $12.272(18)$ & 19091 \\
\hline & & 3.80 & 0.16 & 00 & & $2) \cdot 10^{-2}$ & 5.3510 & 11.463 & 33329 \\
\hline & & 3.82 & 0.16 & 044 & 2 & $\cdot 10^{-2}$ & 5.376 & 10.777 & 22399 \\
\hline & & 3.84 & & & & & & & 20450 \\
\hline & & 3.85 & 0.1 & 62 & 2 & $\cdot 10^{-2}$ & 5.414 & 9.861 & 27895 \\
\hline & & 3.86 & 0.16 & 0.01935 & 262 & 2) $\cdot 10^{-2}$ & 5.4262 & $9.585(14)$ & 24244 \\
\hline & & 3.87 & 0.1 & 909 & & $2.02(2) \cdot 10^{-2}$ & 5.438 & 9.329 & 23330 \\
\hline & & 3.88 & 0.16 & 83 & 271 & $\cdot 10^{-2}$ & 5.4 & 9.0 & 21986 \\
\hline & & 3.90 & 0.16 & & & $2.33(2) \cdot 10^{-2}$ & 5.4731 & 8.653 & 23455 \\
\hline & & 3.92 & 30 & 34 & 288 & $\cdot 10^{-2}$ & 5.4 & 8.25 & 22831 \\
\hline & & 3.94 & 0.1 & & 2 & $2.73(2) \cdot 10^{-2}$ & 5.5 & 7.885 & 8812 \\
\hline & & 3.96 & 93 & 39 & & $2) \cdot 10^{-2}$ & 5.5 & 7.548 & 10974 \\
\hline & & 3.98 & 81 & 14 & & & 2) & & 10889 \\
\hline & & 4.05 & & & & $3.80(2) \cdot 10^{-2}$ & $5.63588(09)$ & & 1418 \\
\hline & & 4.10 & $0.1:$ & 0.01 & 3 & $2) \cdot 10^{-2}$ & $5.68620(11)$ & $5.8511(12)$ & 10233 \\
\hline & & 4.20 & & 0.01261 & 435 & $5.17(2) \cdot 10^{-2}$ & $5.78223(09)$ & $4.9219(05)$ & 10861 \\
\hline & & 4.25 & & & & $\cdot 10^{-2}$ & 5.828 & & 11573 \\
\hline & & 4.35 & $0.1:$ & & 5 & $6.52(2) \cdot 10^{-2}$ & 5.91533(07) & $3.8505(02)$ & 12089 \\
\hline \multirow[t]{8}{*}{6} & 16 & 3.65 & & & & & - & - & 10028 \\
\hline & & 3.70 & 0.1 & 6 & & $6) \cdot 10^{-2}$ & $5.23642(40)$ & $13.341(14)$ & 10198 \\
\hline & & 3.76 & 0.1 & 0.02218 & 29 & $6.44(5) \cdot 10^{-2}$ & $5.31246(25)$ & $11.360(06)$ & 13090 \\
\hline & & 3.80 & 0.16 & 0.02100 & & 4) $\cdot 10^{-2}$ & $5.36159(24)$ & $10.301(05)$ & 11773 \\
\hline & & 3.85 & 0.1 & 22 & 3 & $5) \cdot 10^{-2}$ & $5.42026(22)$ & $9.187(03)$ & 10516 \\
\hline & & 3.90 & 0.160856 & 0.018 & 37 & 5) $\cdot 10^{-2}$ & $5.47736(19)$ & $8.2499(17)$ & 11207 \\
\hline & & 3.97 & 0.15 & 01666 & & $9.53(5) \cdot 10^{-2}$ & $5.55430(11)$ & $7.1530(06)$ & 11109 \\
\hline & & 4.05 & & 44 & 46 & $1.076(4) \cdot 10^{-1}$ & $5.63774(10)$ & $6.2516(06)$ & 15926 \\
\hline \multirow[t]{2}{*}{6} & 20 & 4.20 & & & 58 & - & 5.783 & $4.8317(04)$ & 7568 \\
\hline & & 35 & 0.1 & 0.0 & 71 & - & $5.91643(14)$ & $3.8220(03)$ & 3881 \\
\hline
\end{tabular}

TABLE VIII: Simulation parameters for the D mass ensembles. 
[1] S. Borsanyi, G. Endrodi, Z. Fodor, A. Jakovac, S. D. Katz, S. Krieg, C. Ratti, and K. K. Szabo, JHEP 1011,077 (2010), 1007.2580.

[2] S. Borsanyi, Z. Fodor, C. Hoelbling, S. D. Katz, S. Krieg, and K. K. Szabo, Phys.Lett. B730, 99 (2014), 1309.5258.

[3] A. Bazavov et al. (HotQCD Collaboration), Phys.Rev. D90, 094503 (2014), 1407.6387.

[4] A. Abdel-Rehim, P. Boucaud, N. Carrasco, A. Deuzeman, P. Dimopoulos, et al., PoS LATTICE2013, 264 (2013), 1311.4522.

[5] A. Ali Khan et al. (CP-PACS collaboration), Phys.Rev. D64, 074510 (2001), hep-lat/0103028.

[6] V. Bornyakov, R. Horsley, S. Morozov, Y. Nakamura, M. Polikarpov, P. Rakow, and G. Schierholz, Phys. Rev. D82, 014504 (2010), 0910.2392.

[7] V. Bornyakov, R. Horsley, Y. Nakamura, M. Polikarpov, P. Rakow, and G. Schierholz, PoS Lattice2010, 170 (2010), 1102.4461.

[8] T. Umeda, S. Aoki, S. Ejiri, T. Hatsuda, K. Kanaya, H. Ohno, and Y. Maezawa (WHOT-QCD Collaboration), Phys.Rev. D85, 094508 (2012), 1202.4719.

[9] S. Borsanyi, Z. Fodor, C. Hoelbling, S. D. Katz, S. Krieg, D. Nogradi, B. C. Toth, K. K. Szabo, and N. Trombitas, PoS LATTICE2011, 209 (2011), 1111.3500.

[10] S. Borsanyi, S. Dürr, Z. Fodor, C. Hoelbling, S. D. Katz, S. Krieg, D. Nogradi, B. C. Toth, N. Trombitas, and K. K. Szabo, JHEP 1208, 126 (2012), 1205.0440.

[11] M. Cheng (RBC Collaboration, HotQCD Collaboration), PoS LATTICE2008, 180 (2008), 0810.1311.

[12] M. Cheng, N. H. Christ, P. Hegde, F. Karsch, M. Li, M. F. Lin, R. D. Mawhinney, D. Renfrew, and P. Vranas, Phys.Rev. D81, 054510 (2010), 0911.3450.

[13] T.-W. Chiu, W.-P. Chen, Y.-C. Chen, H.-Y. Chou, and T.-H. Hsieh (TWQCD), PoS LATTICE2013, 165 (2014), 1311.6220.

[14] V. Bornyakov, P. Buividovich, N. Cundy, O. Kochetkov, and A. Schäfer, Phys.Rev. D90, 034501 (2014), 1312.5628.

[15] C. Ratti, S. Borsanyi, G. Endrodi, Z. Fodor, S. D. Katz, S. Krieg, C. Schroeder, and K. K. Szabo, Nucl.Phys. A904-905, 869c (2013).

[16] A. Bazavov et al. (MILC collaboration), PoS LATTICE2013, 154 (2014), 1312.5011.

[17] F. Burger, G. Hotzel, M. Müller-Preussker, E.-M. Ilgenfritz, and M. P. Lombardo, PoS (LATTICE 2013), 153 (2013), 1311.1631.

[18] C. DeTar and U. Heller, Eur.Phys.J. A41, 405 (2009), 0905.2949.

[19] C. DeTar (2011), contribution to Kyoto workshop on Thermal Quantum Field Theory and its Application, August 28-30, 2010, Soryushiron Kenkyu (Study of Particle Theory), 1101.0208.

[20] L. Levkova, PoS LATTICE2011, 011 (2011), 1201.1516.

[21] O. Philipsen, Prog.Part.Nucl.Phys. 70, 55 (2013), 1207.5999.

[22] P. Petreczky, J.Phys. G39, 093002 (2012), 1203.5320.

[23] M. P. Lombardo, PoS (LATTICE 2012), 016 (2012), 1301.7324.

[24] F. Burger, E.-M. Ilgenfritz, M. Kirchner, M. Lombardo, M. Müller-Preussker, O. Philipsen, C. Urbach, and L. Zeidlewicz, Phys.Rev. D87, 074508 (2013), 1102.4530.

[25] F. Burger, E.-M. Ilgenfritz, M. P. Lombardo, M. Kirchner, M. Müller-Preussker, O. Philipsen, C. Pinke, and L. Zeidlewicz, PoS (LATTICE 2012), 068 (2012), 1212.0982.

[26] B. B. Brandt, A. Francis, H. B. Meyer, O. Philipsen, and H. Wittig, PoS LATTICE2013, 162 (2014), 1310.8326.

[27] C. Bonati, P. de Forcrand, M. D'Elia, O. Philipsen, and F. Sanfilippo, Phys.Rev. D90, 074030 (2014), 1408.5086.

[28] P. Boucaud et al. (ETM collaboration), Comput.Phys.Commun. 179, 695 (2008), 0803.0224.

[29] C. Alexandrou, M. Brinet, J. Carbonell, M. Constantinou, P. Harraud, P. Guichon, K. Jansen, T. Korzec, and M. Papinutto (ETM Collaboration), Phys.Rev. D83, 045010 (2011), 1012.0857.

[30] R. Baron et al. (ETM Collaboration), JHEP 1008, 097 (2010), 0911.5061.

[31] K. Jansen, F. Karbstein, A. Nagy, and M. Wagner (ETM Collaboration), JHEP 1201, 025 (2012), 1110.6859.

[32] M. Cheng, N. Christ, S. Datta, J. van der Heide, C. Jung, et al., Phys.Rev. D77, 014511 (2008), 0710.0354.

[33] K. Chetyrkin and A. Retey, Nucl.Phys. B583, 3 (2000), hep-ph/9910332.

[34] O. Philipsen and L. Zeidlewicz, Phys. Rev. D81, 077501 (2010), 0812.1177.

[35] F. Karsch, E. Laermann, and A. Peikert, Phys.Lett. B478, 447 (2000), hep-lat/0002003.

[36] M. Cheng, S. Ejiri, P. Hegde, F. Karsch, O. Kaczmarek, et al., Phys.Rev. D81, 054504 (2010), 0911.2215.

[37] A. Bazavov, T. Bhattacharya, M. Cheng, C. DeTar, H. Ding, et al., Phys.Rev. D85, 054503 (2012), 1111.1710.

[38] K. Olive et al. (Particle Data Group), Chin.Phys. C38, 090001 (2014).

[39] P. Huovinen and P. Petreczky, Nucl.Phys. A837, 26 (2010), 0912.2541.

[40] K. Jansen, C. McNeile, C. Michael, and C. Urbach (ETM Collaboration), Phys.Rev. D80, 054510 (2009), 0906.4720.

[41] K. Jansen, C. Michael, and C. Urbach (ETM Collaboration), Eur.Phys.J. C58, 261 (2008), 0804.3871.

[42] C. Alexandrou, R. Baron, J. Carbonell, V. Drach, P. Guichon, K. Jansen, T. Korzec, and O. Pène (ETM Collaboration), Phys.Rev. D80, 114503 (2009), 0910.2419.

[43] C. McNeile, C. Michael, and C. Urbach (ETM Collaboration), Phys.Lett. B674, 286 (2009), 0902.3897.

[44] G. Boyd, J. Engels, F. Karsch, E. Laermann, C. Legeland, M. Lütgemeier, and B. Petersson, Nucl.Phys. B469, 419 
(1996), hep-lat/9602007.

[45] S. Borsanyi, G. Endrodi, Z. Fodor, S. Katz, and K. Szabo, JHEP 1207, 056 (2012), 1204.6184.

[46] J. Liao and E. Shuryak, Phys.Rev.Lett. 109, 152001 (2012), 1206.3989.

[47] K. Miura and M. P. Lombardo, Nucl.Phys. B871, 52 (2013), 1212.0955.

[48] K. Jansen and C. Urbach, Comput.Phys.Commun. 180, 2717 (2009), 0905.3331.

[49] A. Abdel-Rehim, F. Burger, A. Deuzeman, K. Jansen, B. Kostrzewa, L. Scorzato, and C. Urbach, PoS LATTICE2013, 414 (2014), 1311.5495.

[50] R Development Core Team, R: A language and environment for statistical computing, R Foundation for Statistical Computing, Vienna, Austria (2010), ISBN 3-900051-07-0, URL http://www.R-project.org.

[51] F. Burger, G. Hotzel, K. Jansen, and M. Petschlies (2014), 1412.0546. 